\title{
The blood-brain barrier: an engineering perspective
}

\section{Andrew D. Wong ${ }^{1,2}$, Mao Ye ${ }^{1,2}$, Amanda F Levy ${ }^{1,2}$, Jeffrey D. Rothstein ${ }^{3,4}$, Dwight E. Bergles ${ }^{3}$ and Peter C. Searson ${ }^{1,2 *}$}

${ }^{1}$ Department of Materials Science and Engineering, Johns Hopkins University, Baltimore, MD, USA

2 Institute for Nanobiotechnology, Johns Hopkins University, Baltimore, MD, USA

${ }^{3}$ The Solomon H. Snyder Department of Neuroscience, Johns Hopkins University, Baltimore, MD, USA

${ }^{4}$ Brain Sciences Institute, Johns Hopkins University, Baltimore, MD, USA

\section{Edited by:}

Jay Nadeau, McGill University,

Canada

Reviewed by:

Hari S. Sharma, Uppsala University, Sweden

Antonio Malgaroli, Vita-Salute San

Raffaele University, Italy

\section{*Correspondence:}

Peter C. Searson, Institute for Nanobiotechnology, Johns Hopkins University, 120 Croft Hall, 3400

North Charles Street, Baltimore,

MD 21218, USA

e-mail: searson@jhu.edu
It has been more than 100 years since Paul Ehrlich reported that various water-soluble dyes injected into the circulation did not enter the brain. Since Ehrlich's first experiments, only a small number of molecules, such as alcohol and caffeine have been found to cross the blood-brain barrier, and this selective permeability remains the major roadblock to treatment of many central nervous system diseases. At the same time, many central nervous system diseases are associated with disruption of the blood-brain barrier that can lead to changes in permeability, modulation of immune cell transport, and trafficking of pathogens into the brain. Therefore, advances in our understanding of the structure and function of the blood-brain barrier are key to developing effective treatments for a wide range of central nervous system diseases. Over the past 10 years it has become recognized that the blood-brain barrier is a complex, dynamic system that involves biomechanical and biochemical signaling between the vascular system and the brain. Here we reconstruct the structure, function, and transport properties of the blood-brain barrier from an engineering perspective. New insight into the physics of the blood-brain barrier could ultimately lead to clinical advances in the treatment of central nervous system diseases.

Keywords: blood-brain barrier, neurovascular unit, capillary, microvasculature, transport

\section{THE PHYSICS OF THE BLOOD-BRAIN BARRIER (BBB) POWERING THE BRAIN}

The BBB is the interface between the vascular system and the brain, and hence we begin by reviewing the architecture of the brain vasculature. The human brain is comprised of $\sim 100$ billion neurons and consumes about $15-20 \mathrm{~W}$ power. The metabolic nutrients that supply the power are oxygen and glucose. The brain, along with the liver and GI tract, are the most energy expensive organs in the human body. Overall the brain accounts for $15-20 \%$ of the base metabolic rate (BMR), consuming $15-20 \%$ of oxygen leaving the heart, and $15-20 \%$ of the glucose consumed daily (Aiello and Wheeler, 1995; Attwell and Laughlin, 2001; Fish and Lockwood, 2003; Lennie, 2003; Navarrete et al., 2011). Since the brain does not have significant capacity to store metabolic nutrients, fuel to power the brain is provided ondemand by the lungs, and GI system which transfer oxygen and glucose, respectively, to the vascular system. Therefore, the role of the vascular system is crucial in delivering nutrients necessary to maintain normal brain function. Interruption of cerebral blood flow very quickly results in neuronal death; after cardiac arrest apoptosis of neurons begins almost immediately, and brain damage occurs after about 5 min (Hossmann, 2006).

Most of the energy consumed by ATP hydrolysis in the brain is used by neurons for generating nerve impulses (e.g., voltageand ligand-gated ion channels) and for maintaining ion gradients (e.g., sodium/potassium pumps; Attwell and Laughlin, 2001; Shulman et al., 2004; Raichle and Mintun, 2006). The cerebral metabolic rate (CMR) for ATP utilization in the human brain is about $9.5 \mu \mathrm{mol} \mathrm{g}-1 \mathrm{~s}^{-1}$ in gray matter and about $3 \mu \mathrm{mol}$ $\mathrm{g}^{-1} \mathrm{~s}^{-1}$ in white matter (Zhu et al., 2012). Therefore, about $77 \%$ of the brain's energy consumption is in cortical gray matter, which represents about $50 \%$ of the brain volume (Zhu et al., 2012). The gray matter consists of neurons, dendrites, unmyelinated axons, glial cells, and capillaries, whereas white matter is mostly myelinated axons, glial cells, and capillaries. Due to the increased energy demands, the capillary density is $2-4$ times higher in gray matter (Borowsky and Collins, 1989b; Heinzer et al., 2008). Gray matter has a higher density of synapses and higher levels of neural activity than white matter and hence increased energy consumption is expected (Zhu et al., 2012). As we show below, the architecture of the brain microvasculature is dictated in large part by the energy needs of the neurons in the brain.

\section{BRAIN MICROVASCULATURE ACROSS SPECIES}

The architecture of the brain microvasculature across species is remarkably similar. The BMR of numerous species (both warm blooded and cold-blooded organisms) follows Keliber's law where the BMR is proportional to body weight with an exponent of 0.75 (Kleiber, 1947). The oxygen $\left(\mathrm{mL} \mathrm{min}^{-1}\right)$ and glucose $(\mu \mathrm{mol}$ $\mathrm{min}^{-1}$ ) CMRs increase with brain volume across species, with an exponent of 5/6 (0.85), indicating that the brain is a major energy consumer (Karbowski, 2007, 2009, 2011). The metabolic rate of the human brain, normalized to its mass is about $11 \mathrm{~W} \mathrm{~kg}^{-1}$, 
almost an order of magnitude larger than that of the human body of $1.3 \mathrm{~W} \mathrm{~kg}^{-1}$ (Aiello and Wheeler, 1995).

From the exponent of 5/6, it follows that the CMR normalized by brain volume scales with brain volume $(V)$ with an exponent of $-1 / 6\left(\mathrm{CMR} / V \propto V^{-1 / 6}\right.$; Karbowski, 2011). Cerebral blood flow, normalized to brain volume, also has an exponent of $-1 / 6$ $\left(\mathrm{CBF} / V \propto V^{-1 / 6} ;\right.$ Karbowski, 2011), illustrating that cerebral blood flow is directly proportional to CMR across species (CMR $\propto \mathrm{CBF})$. Indeed, studies in rat brains have shown a strong correlation between local blood flow, glucose utilization, and capillary density (Klein et al., 1986; Borowsky and Collins, 1989a).

The neuron density in the brain scales with brain volume with an exponent of $-1 / 6\left(\rho_{n} \propto V^{-1 / 6}\right.$; Karbowski, 2011). The negative exponent reflects the difficulty in wiring and powering $3 \mathrm{D}$ architectures with increasing brain size. The capillary length density also exhibits an exponent of $-1 / 6\left(\rho_{c} \propto V^{-1 / 6}\right)$ showing that the number of neurons is proportional to the total length of capillaries across species (Karbowski, 2011). Equivalently, the capillary length density per neuron is constant across species. The average capillary diameter is only weakly dependent on brain volume with an exponent of $0.08\left(d_{c} \propto V^{0.08}\right)$, increasing from about $4 \mu \mathrm{m}$ in the rat brain to about $7 \mu \mathrm{m}$ in the human brain.

The fact that cerebral blood flow is directly proportional to CMR and that the capillary length density per neuron is constant across species provides evidence that the architecture of the human brain microvasculature is not unique. Neural function is constrained in part by energy demands and hence the spatial distribution of capillaries is closely correlated with metabolic function.

\section{MICROVASCULATURE OF THE HUMAN BRAIN}

The average adult human brain weighs about $1500 \mathrm{~g}$ and occupies about $1200 \mathrm{~cm}^{3}$. The surface area of microvessels is $100-200 \mathrm{~cm}^{2}$ $\mathrm{g}^{-1}$ tissue (Crone, 1963; Gross et al., 1986), corresponding to a total surface area of $15-25 \mathrm{~m}^{2}$. In comparison, the surface area of the gut is $300-400 \mathrm{~m}^{2}$, the lung is about $100 \mathrm{~m}^{2}$, and the skin is about $2 \mathrm{~m}^{2}$. The microvessel density is about $500 \mathrm{~m}$ $\mathrm{cm}^{-3}$ (Kreczmanski et al., 2005, 2009), corresponding to a total microvessel length of about $600 \mathrm{~km}$ in the adult human brain (Zlokovic, 2005).

The human brain is comprised of about 100 billion neurons and a similar number of glial cells. Neurons, astrocytes, microglia, and pericytes account for almost $80 \%$ of the brain volume. The extracellular space occupies $15-30 \%$ of the brain volume (Nicholson, 2001) and the brain vasculature about 3\% of the brain volume.(Nicholson, 2001) Capillaries in the brain may be as small as $7-10 \mu \mathrm{m}$ in diameter and the average intercapillary distance is about $40 \mu \mathrm{m}$ (Duvernoy et al., 1983; Nicholson, 2001). Consequently, the cell body of a neuron is typically about 10-20 $\mu \mathrm{m}$ from the nearest capillary (Schlageter et al., 1999).

Blood is supplied to the brain through four arteries, the internal carotid arteries and the vertebral arteries, which merge in the circle of Willis at the base of the brain (Hossmann, 2006). Each carotid artery supplies about $40 \%$ of the total blood flow to the brain. The flow rate to the brain is about $800 \mathrm{~mL} \mathrm{~min}^{-1}$ (Zlokovic, 2005), about 15-20\% of the total blood flow from the heart. From the circle of Willis, intercerebral arteries, and pial arteries are distributed along the surface of the brain, from which arteries and arterioles penetrate into the brain parenchyma perpendicular to the brain surface, leading to the network of capillaries. In the human brain, capillaries form numerous connections before merging into venules and veins. Blood flow exits the brain through the jugular veins. While the arteries and arterioles are sheathed in one or more layers of smooth muscle cells, the capillaries are surrounded by pericytes and astrocytes.

The architecture of the vasculature can be described in terms of individual capillary segments between two junctions. The capillary length density $\rho_{c}=N_{c} \mathrm{~L}_{c} / V$ where $N_{c}$ is the number of segments of average length $L_{c}$. Typical segment lengths in a mouse cortex are about $60 \mu \mathrm{m}$, with segment densities and junction densities of about $10,000 \mathrm{~mm}^{-3}$ (Heinzer et al., 2008). The tortuosity ( $\tau$ ) of a segment is given by $\tau=$ $\lambda / c$, where $\lambda$ is the length of a segment and $c$ is the chord length. Typical values for tortuosity in the mouse cortex are 1.2-1.3 (Heinzer et al., 2008). The number of segments connected at a junction $n \approx 3.5$, indicating that a significant fraction of junctions are higher order than a simple bifurcation $(n=3)$.

Blood flow and heart rate are regulated by the autonomic nervous system, located in the medulla in the lower midbrain. The medulla receives sensory input from other brain regions and stimulates cardiovascular responses through nerve fibers that travel to the heart and blood vessels. Varicosities along the fibers are the sites for release of neurotransmitters. Autonomic and sensory nerve fibers are associated with the cerebral arteries, pial arteries, and arterioles in the brain, and release neurotransmitters such as norepinephrine (NE) and neuropeptide Y (NPY) that result in vessel constriction, and acetylcholine (Ach) and vasoactive intestinal polypeptide (VIP) that can dilate vessels. Regulation of brain capillaries by the autonomic nervous system has not been established, however, pericytes can dilate and contract in response to different neurotransmitters suggesting the possibility of autonomous signaling (Peppiatt et al., 2006; Fisher, 2009; Fernandez-Klett et al., 2010; Krueger and Bechmann, 2010).

Over the last 3 million years, from Australopithecus to Homo sapiens, the size of the human brain has increased from about $400 \mathrm{~cm}^{3}$ to about $1200 \mathrm{~cm}^{3}$ in modern humans (Aiello and Dunbar, 1993; Potts, 2011). This increase has not been continuous but has had several periods of rapid expansion. The expensive tissue hypothesis postulates that the increase in power consumption associated with increasing brain size must be balanced by a decrease in the power requirements in the liver and GI tract (Aiello and Wheeler, 1995). However, recent evidence suggests that these evolutionary increases in brain size are related to an increase in energy input, such as improved diet and availability of food, and changes in energy allocation, such as decreased energy costs associated with locomotion (Holliday, 1986; Roth and Dicke, 2005; Navarrete et al., 2011). During prenatal and early childhood development, the developing brain consumes $60 \%$ or more of the basal metabolism, and it has been argued that this is a fundamental limitation to brain size in humans (Snodgrass et al., 2009). 
The microvasculature in the brain differs from other capillary networks in the human body, for example those in the lung, in two significant ways. First, the brain microvasculature tightly regulates transport into the brain. Second, the capillaries can exhibit significant plasticity in response to abnormal physiological conditions. For example, during ischemia capillaries can dilate to increase oxygen influx (Boero et al., 1999; Ito et al., 2003; Hauck et al., 2004).

The architecture of the brain microvasculature is very similar across species, indicating that the human brain microvasculature is not physically unique. However, an important question is whether the human blood-brain barrier is functionally different from other species. Evidence suggests that there may be significant biochemical differences, for example in the expression levels of transporters and pumps that make the human blood-brain barrier unique (Hammarlund-Udenaes et al., 2008). However, the evolutionary pressures that influenced these differences remains to be established. Elucidating these differences is key in studies of central nervous system diseases and in developing drug therapies.

\section{MAINTAINING BRAIN HOMEOSTASIS}

The supply of metabolic nutrients to the human brain is achieved through a network of over $600 \mathrm{~km}$ of small capillaries, about $7 \mu \mathrm{m}$ in diameter, such that each neuron is within $20 \mu \mathrm{m}$ of a capillary. The drawback of this architecture is that the brain requires a tightly regulated local environment for cells to function. Since the brain microvasculature has a very large surface area $\left(15-25 \mathrm{~m}^{2}\right)$, maintaining homeostasis and preventing interference with signal generation and transmission in is a major challenge.

The blood-brain barrier is responsible for maintaining homeostasis of the brain by regulating the chemical environment, immune cell transport, and the entry of xenobiotics (Hawkins and Davis, 2005; Abbott et al., 2010). The concentrations of water, ions, amino acids, hormones, and neurotransmitters in the blood undergo fluctuations, particularly after eating or exercise. If such fluctuations were allowed to occur in the brain it would lead to local disruption of signal propagation and uncontrolled neural activity, and hence transport from the capillary lumen to the brain parenchyma must be tightly regulated. Immune cell transport (e.g., leukocytes) must also be regulated as the brain is contained in a fixed volume in the skull and an inflammatory response could lead to an increase in intercranial pressure or cerebral edema. Finally, the entry of toxins and pathogens, such as bacteria and viruses circulating in the blood, can lead to neuron cell death and hence must also be prevented (Begley and Brightman, 2003; Hawkins and Davis, 2005; Abbott et al., 2010).

The tight junctions formed by brain microvascular endothelial cells (BMECs) regulate paracellular transport whereas transcellular transport is regulated by specialized transporters, pumps, and receptors (Figure 1) (Chishty et al., 2001; Demeule et al., 2002; Hawkins et al., 2002; Ohtsuki and Terasaki, 2007; Ueno, 2009; Abbott et al., 2010; Hartz and Bauer, 2011).

\section{CIRCUMVENTRICULAR ORGANS}

While the blood-brain barrier maintains homeostasis, there are specialized regions of the brain that allow direct communication
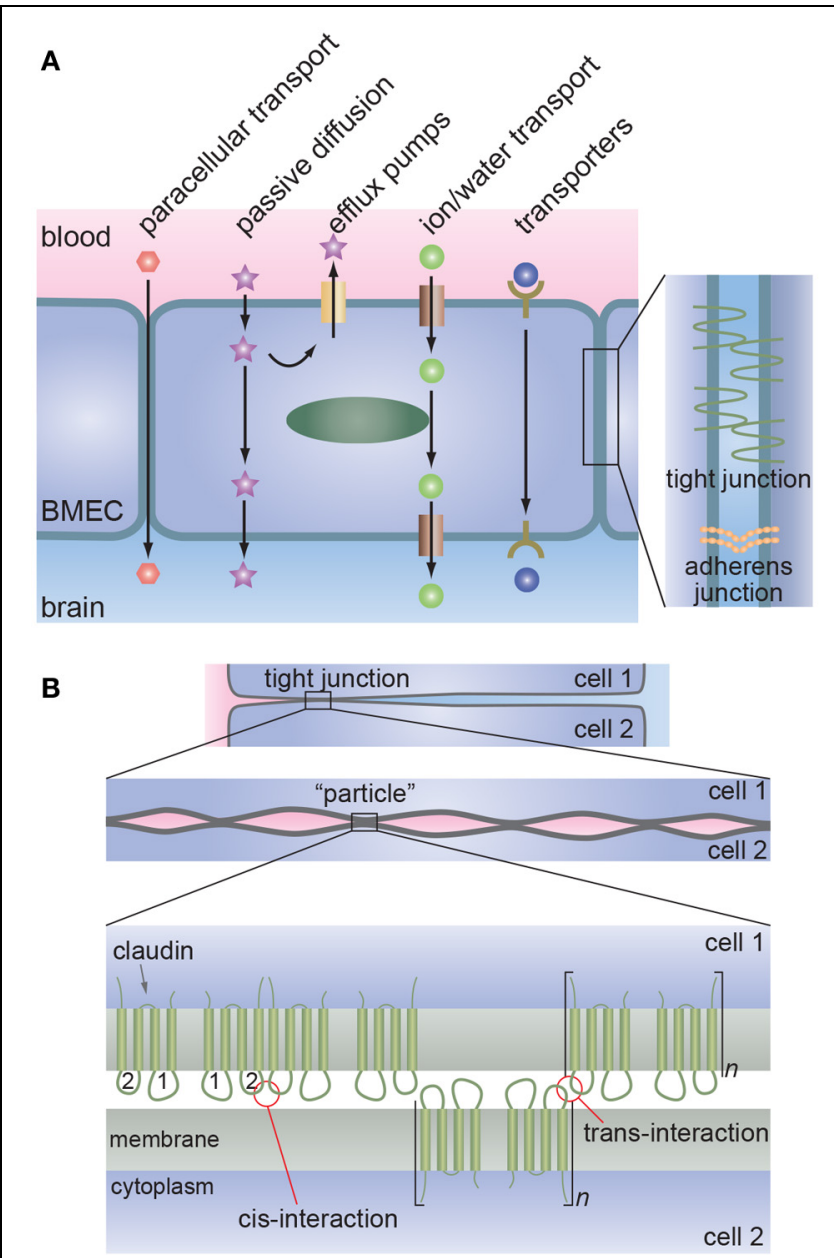

FIGURE 1 | The blood-brain barrier. (A) Schematic illustration of transport across the brain microvascular endothelial cells (BMECs) that form the lumen of brain capillaries. Paracellular transport is severely restricted due to the formation of tight junctions between endothelial cells. Metabolic nutrients and other essential molecules are transported across the luminal and abluminal membranes by channels, pumps, or mediated transport systems. Small lipophilic molecules can passively diffuse across the lipid bilayer but, in many cases, are returned to the blood by efflux pumps. (B) Proposed molecular interactions at tight junctions. Lateral association of claudins (cis-interaction) results in the formation of oligomers whereas association of claudins on opposing membranes (trans-interaction) results in tight junction formation. Multiple regions of trans-interactions appear as particles in electron microscopy images.

between the brain and the vascular system. In the circumventricular organs, located at the surface of the third and fourth ventricles, the blood-brain barrier is more permeable. Neurons and glial cells at these sites can sense changes in the concentration of various molecules, such as hormones, and secrete hormones, neurotransmitters, or cytokines into the circulation (Ganong, 2000; Duvernoy and Risold, 2007; Benarroch, 2011). These organs include: the neurohypophysis (posterior pituitary), the median eminence, the area postrema (vomiting center), the subfornical organ, and the vascular organ of the lamina terminalis. 


\section{HISTORY}

In 1885, Paul Ehrlich reported that various water-soluble dyes injected into the circulatory system did not stain the brain and spinal cord, and hypothesized that the CNS had a lower affinity for these dyes (Ribatti et al., 2006; Liddelow, 2011). In 1898, Biedl and Kraus showed that cholic acids (bile acids) that induce seizures and coma when injected into the brain, were not toxic when injected into the circulatory system (Ribatti et al., 2006). In 1900, Lewandowsky reported similar findings with potassium ferrocyanide and also concluded that there was limited permeation from the circulatory system into the brain (Ribatti et al., 2006), a phenomenon to which he ascribed the term bluthirnschranke (blood-brain barrier). Later, Ehrlich's student Edwin Goldmann showed that the water-soluble dye trypan blue $(M W=960.8)$ injected into cerebrospinal fluid readily stained central nervous tissue blue, contradicting Ehrlich's conclusion of a lower binding affinity of the central nervous system for these dyes, and supporting the hypothesis of limited permeation from the circulatory system into the brain (Ribatti et al., 2006). In 1967, Reese and Karnovsky used high resolution electron microscopy to demonstrate that horseradish peroxidase (HRP, $40 \mathrm{kDa}$ ) was prevented from entering the CNS by tight junctions (Bradbury, 1993). They showed that the tight junctions were continuous and concluded that the blood brain barrier existed at the level of the vascular endothelium.

\section{THE NEUROVASCULAR UNIT INTRODUCTION}

Historically, the blood-brain barrier has been defined by the layer of endothelial cells that form the vessel/capillary walls. More recently, the concept of the neurovascular unit has been introduced to recognize that brain health depends on functional interactions between neurons and non-neuronal cells such as vascular cells (endothelial cells and pericytes) and glia (astrocytes, microglia, and oligodendroglia; Figure 2) (Hawkins and Davis, 2005; Abbott et al., 2010). This is a highly dynamic system in which cells transduce biochemical and biomechanical signals in complex microenvironments involving basement membrane and extracellular matrix. These non-neuronal cells are responsible for the physical, biochemical, and immune barriers of the CNS that regulate the microenvironment of the neurons which is key for signal transduction, remodeling, angiogenesis, and neurogenesis.

\section{ENDOTHELIAL CELLS}

The endothelial cells that line the microvasculature in the brain define the interface between the vascular system and the brain. These cells function as adaptive non-linear input/output devices where input from biomechanical and biochemical forces in the local microenvironment of the neurovascular unit influences cell phenotype as manifested by cell morphology, protein expression, gene expression, proliferation, transport, etc. (Dejana, 2004; Aird, 2005, 2007). In addition to biochemical and biomechanical input from the vascular system, numerous paracrine signaling pathways between microvascular endothelial cells and astrocytes and pericytes are responsible for maintenance of the blood-brain barrier (Aird, 2007; Abbott et al., 2010).

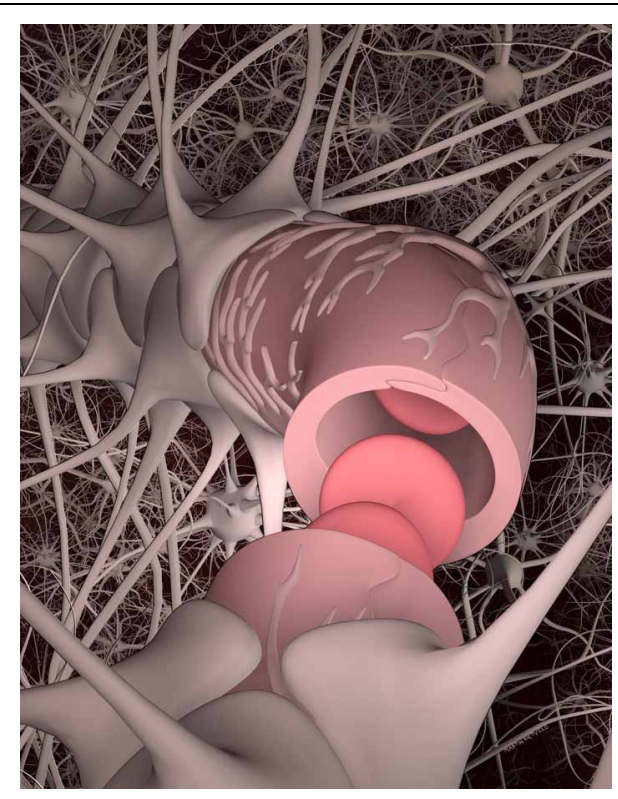

FIGURE 2 | The neurovascular unit. The microvascular endothelial cells that form the lumen of brain capillaries are partially covered by pericytes and basement membrane, and almost completely surrounded by the end feet of astrocytes. Functional interactions between BMECs, astrocytes, pericytes, other glial cells, and neurons are key to regulating brain homeostasis. Blood flow is associated both biomechanical and biochemical signaling mediated by multiple cell types and soluble factors. The brain microvascular endothelial cells function in a cylindrical geometry with high curvature and experience shear stress resulting from blood flow. The BMECs and pericytes are surrounded by basement membrane consisting primarily of fibronectin, laminin 1, and collagen type IV. The extra-cellular matrix in the brain is based on hyaluronic acid.

In the brain microvasculature, cell-cell junctions are key to maintaining the integrity of the brain microvasculature and regulating paracellular transport. Cell-cell adhesion is achieved through the formation of adherens junctions and tight junctions (Bazzoni and Dejana, 2004; Dejana, 2004; Aird, 2007). Both adherens junctions and tight junctions involve homophilic interactions between the extracellular domains of membrane proteins and are linked to the actin cytoskeleton via intracellular partners. Endothelial adherens junctions are formed by the extracellular domains of vascular endothelial cadherin (VE-cadherin) and are linked to the actin cytoskeleton inside the cell via proteins such as $\alpha$-catenin, $\beta$-catenin, and vinculin (Bazzoni and Dejana, 2004; Dejana, 2004).

The tight junctions in the brain microvasculature prevent paracellular transport of most molecules and severely restrict transport of small ions. Therefore, transcellular transport is responsible for most molecular trafficking between the vascular system and the brain. Various methods for transient disruption of tight junctions have been explored for drug delivery, and local disruption of tight junctions is associated with many diseases of the central nervous system.

Tight junctions are formed between claudins (Nitta et al., 2003), although other proteins such as occludin are also present (Hawkins and Davis, 2005; Furuse and Tsukita, 2006). These tight 
junction membrane proteins are connected to the actin cytoskeleton via zona occludin ( $\mathrm{ZO}$ ) adaptor molecules (ZO-1 and ZO-2; Hawkins and Davis, 2005). The claudin family consists of more than 20 proteins that are essential for the formation of tight junctions. Claudin-5 is the isoform most commonly found in the BBB (Morita et al., 1999; Nitta et al., 2003; Hewitt et al., 2006), although claudin-1 and claudin-12 are also associated with tight junction formation (Wolburg and Lippoldt, 2002; Dejana, 2004; Abbott et al., 2006). Antibodies to claudin-5, occludin, and ZO1 are commonly used as markers of tight junction formation in monolayers of BMECs (Cecchelli et al., 2007). Adherens junctions and tight junctions are structurally and functionally linked. For example, evidence suggests that VE-cadherin at adherens junctions upregulates the gene encoding the tight junction protein claudin-5 (Dejana, 2004; Taddei et al., 2008).

In high resolution electron microscope images, tight junctions appear as a series of discrete sites of apparent fusion near the apical surface (Reese and Karnovsky, 1967; Brightman, 1977). These sites are often described as strands of "particles" along the junction (Figure 2) (Tsukita and Furuse, 1999; Tsukita et al., 2001); the backbone of these strands is composed of claudins.

The claudins have a molecular weight of about $23 \mathrm{kDa}$ and have four transmembrane segments, one intracellular loop, Nand C-terminal cytoplasmic domains, and two extra cellular loops (Furuse and Tsukita, 2006; Krause et al., 2009). The first extracellular loop ECL1 consists of about 50 amino acids whereas ECL2 consists of about 25 amino acids (Krause et al., 2009). Both of the extra-cellular loops (ECL1 and ECL2) of claudin-5 are thought to play a role in tight junction formation (Krause et al., 2009). Claudin-5 forms oligomers in one membrane via cis-interactions between ECL2s (Piontek et al., 2008). Trans-interactions between ECL2s on opposing membranes result in polymerization and formation of the particles observed in electron microscopy. (Piontek et al., 2008) Mutations of the two cysteines in claudin-5 have been shown to reduce barrier properties, suggesting that these two residues are important in tight junction formation (Wen et al., 2004).

The morphology of microvascular endothelial cells is dependent in part on biomechanical input from the vascular system. The shear stress associated with blood flow results in elongation and alignment of endothelial cells in the direction of flow (Caplan et al., 1974; Nerem et al., 1981; Ohashi and Sato, 2005; Aird, 2007). In vitro studies have confirmed that the elongation and alignment of endothelial cells in a 2D confluent monolayer increases with increasing shear stress (Levesque and Nerem, 1985; Malek and Izumo, 1996). In large vessels, there are many cells around the perimeter, however, in small capillaries endothelial cells can wrap around to form tight junctions with themselves, as well as their neighbors (Nag, 2003). Shear stress can also upregulate genes associated with junctional proteins and transporters (Cucullo et al., 2011).

The turnover of BMECs, measured as the mitotic index or the turnover time, is thought to be very low (Hobson and Denekamp, 1984; Ekstrand et al., 2008). However, most studies are based on extrapolation from relatively short intervals. The activation and increase in turnover of BMECs due to angiogenesis and vascular remodeling is also unknown. Similarly, the response of a capillary to endothelial cell apoptosis or disruption is not wellunderstood. Endothelial progenitor cells from bone marrow may be involved in repair of the blood-brain barrier however, the signaling processes involved in recruiting these cells and initiating differentiation are not known (Asahara et al., 2011).

\section{ASTROCYTES}

Astrocytes are involved in multiple processes in the brain, including regulation of ion and water concentration, the clearance of neurotransmitters, proliferation of stem cells, control of the number of synapses, and maintenance of the BBB (Ullian et al., 2001; Lee et al., 2003; Volterra and Meldolesi, 2005; Abbott et al., 2006; Fiacco et al., 2009; Freeman, 2010; Halassa and Haydon, 2010). Morphologically, astrocytes are usually star shaped with many processes or protrusions emanating from the cell body, with an overall diameter of about $140 \mu \mathrm{m}$ in the human brain (Oberheim et al., 2009). Astrocytes interact with microvascular endothelial cells through the end-feet of the protrusions that wrap around the capillary (Abbott, 2002; Abbott et al., 2006). Brain capillaries are often almost completely surrounded by astrocytic end-feet and one astrocyte may contact multiple capillaries (Oberheim et al., 2009). Astrocytes form contacts with microvessels and the synapses between neurons, and play an important role in matching oxygen and glucose transport to neural activity through regulation of local blood flow (Zonta et al., 2003; Iadecola, 2004; Takano et al., 2006; Iadecola and Nedergaard, 2007). Evidence suggests that intracellular $\mathrm{Ca}^{2+}$ is involved in blood flow regulation since neuronal stimulation results in an elevation of intracellular $\mathrm{Ca}^{2+}$ concentration in astrocyte end-feet (Iadecola, 2004).

Astrocytes participate in the formation of the BBB by enhancing tight junction formation, modulating the expression and polarization of transporters, and promoting specialized enzyme systems (debault and Cancilla, 1980; Janzer and Raff, 1987; Abbott, 2002; Lee et al., 2003; Haseloff et al., 2005; Abbott et al., 2006). Several astrocyte derived factors, including glialderived neurotrophic factor (GDNF), basic fibroblast growth factor (BFGF), and angiopoetin-1 (ANG-1) are known to induce blood-brain barrier characteristics in endothelial cells (Haseloff et al., 2005; Abbott et al., 2006).

\section{PERICYTES}

Pericytes wrap around microvessels and capillaries in the brain (Sims, 1986; Fisher, 2009; Attwell et al., 2010; Krueger and Bechmann, 2010; Dalkara et al., 2011; Winkler et al., 2011) and communicate with endothelial cells, astrocytes, and neurons in the neurovascular unit (Bonkowski et al., 2011). Morphologically, pericytes tend to be aligned with the vessel axis and extend protrusions that wrap around the capillaries (Bonkowski et al., 2011). A thin layer of basement membrane separates pericytes from endothelial cells, and from surrounding astrocyte end-feet. The ratio of pericytes to endothelial cells is typically around 1:3 (Shepro and Morel, 1993). Direct peg-and-socket contacts that span the intervening basement membrane and gap junctions with endothelial cells initiate multiple signaling pathways (Bonkowski et al., 2011). For example, platelet-derived growth factor B (PDGF-B) on endothelial cells binds with the corresponding 
receptor (PDGFR-B) on pericytes, regulating recruitment of pericytes as well as their proliferation (Bell et al., 2010; Dalkara et al., 2011; Winkler et al., 2011).

Pericytes are contractile, with actin stress fibers throughout the cell body, and contribute to the regulation of blood flow by controlling capillary diameter (Peppiatt et al., 2006; Hamilton et al., 2010; Dalkara et al., 2011). In cell culture, pericytes are usually identified by $\alpha$-smooth muscle actin, which is not expressed in endothelial cells, although expression can be heterogeneous.(Dalkara et al., 2011) Pericytes do not express GFAP, expressed by astrocytes, or vWF, expressed by ECs.

Studies in mice have shown that perictyes are recruited to nascent capillaries during development (Daneman et al., 2009, 2010) and are key for development of the BBB and regulating transport across the BBB (Armulik et al., 2005, 2010; Daneman et al., 2009, 2010; Kim et al., 2009) Indeed, pericyte loss leads to locally reduced cerebral blood flow and breakdown of the blood-brain barrier (Armulik et al., 2010; Bell et al., 2010).

Pericytes are able to either enhance or impair blood brain barrier function in in vitro models depending on their state of differentiation. Pericytes differentiated with TGF $\beta$ are $\alpha$ actin positive and have been found to decrease transendothelial electrical resistance (TEER), while pericytes differentiated with BFGF are $\alpha$-actin negative and increase TEER above controls (Thanabalasundaram et al., 2011).

\section{CELL LINES FOR RESEARCH}

A major challenge for the development of in vitro models of the BBB is the availability of appropriate cell lines, particularly BMECs. An in vitro model of the human BBB should exhibit restricted paracellular transport $\left(\right.$ TEER $=1 \mathrm{k} \Omega \mathrm{cm}^{2}, P_{\text {sucrose }}<$ $10^{-7} \mathrm{~cm} \mathrm{~s}^{-1}$ ), BMECs with the morphology and characteristics typical of the BBB, expression of BBB-specific markers and transporters, and be readily available, convenient to use, and reproducible (Reichel et al., 2003). While primary human BMECs are often considered preferable for in vitro models, the difficulties in harvesting and purification of these cells can significantly limit accessibility and reliability (Stins et al., 1997; Bernas et al., 2010). In general, primary cells are used only at very low passage numbers to avoid down-regulation of BBB characteristics (Reichel et al., 2003). In contrast, currently available cell lines can overcome limitations associated with accessibility and convenience, but do not exhibit all of the required features of the human BBB (Sloan et al., 2012). Nevertheless, specific cell lines may recapitulate properties that are necessary for some physiological, pathological, or pharmacological applications. Common sources for animal BMECs include rodent, bovine, and porcine brain cortices. Primary astrocytes and pericytes can also be extracted from the brain cortex (Siddharthan et al., 2007). Advances in stem cell engineering suggest that differentiation of stem cells to BMECs (Lippmann et al., 2012) and astrocytes (Krencik et al., 2011) may ultimately solve the problem of limited cell lines.

\section{BASAL LAMINA AND ECM}

The basement membrane surrounding the endothelial cells and pericytes is comprised of fibronectin, laminin (411, 421, and 511) (Aumailley et al., 2005), and collagen type IV (Tilling et al.,
1998, 2002; Hartmann et al., 2007). The thickness of the basement membrane, determined from electron microscope images, is about $100 \mathrm{~nm}$ (Nag, 2003). Endothelial cell monolayers on fibronectin, laminin 1, and collagen type IV show enhanced TEER, suggesting a role for the basement membrane in enhancing the formation of tight junctions (Tilling et al., 1998, 2002; Hartmann et al., 2007).

The extracellular matrix in the brain is composed of four main components: hyaluronic acid (HA), lecticans, hyaluronan and proteoglycan link proteins (HAPLNs), and tenascins (Zimmermann and Dours-Zimmermann, 2008). Common ECM proteins such as fibronectin and collagen type I are not present in the brain (Sanes, 1989). HA is a long unbranched polysaccharide with negatively charged disaccharide repeat units, and is unique amongst the glycosaminoglycans (GAGs) in that it is nonsulfated (Laurent and Fraser, 1992; Toole, 2004; Zimmermann and Dours-Zimmermann, 2008; Ananthanarayanan et al., 2011). HA is synthesized by hyaluronan synthases at the inner surface of the plasma membrane, and can have a molecular weight as high as $10^{7} \mathrm{Da}$ (Zimmermann and Dours-Zimmermann, 2008). HA can interact with cells through binding to cell surface receptors, such as CD44 and RHAMM (Turley et al., 2002). The lecticans are a family of chondroitin sulfate proteoglycans with an HA binding domain and include aggrecan, versican, neurocan, and brevican (Yamaguchi, 2000). The tenascins (Tns) are large multimeric glycoproteins. Tn-C and Tn- $\mathrm{R}$ are thought to bind to multiple lecticans and link proteins thereby crosslinking the HA and forming a 3D network (Zimmermann and Dours-Zimmermann, 2008).

The interstitial fluid in the brain is similar in composition to blood plasma, however, it has lower $\mathrm{K}^{+}$and $\mathrm{Ca}^{2+}$ concentrations but higher $\mathrm{Mg}^{2+}$ concentration. In addition, the interstitial fluid has a lower protein content than plasma.

\section{EXTRACELLULAR SPACE (ECS)}

The ECS is the region between cells in the brain and provides the main pathway for transport between capillaries and neurons and other cells in the brain. Although most neurons are within $10-20 \mu \mathrm{m}$ of a capillary, transport in the extracellular space is usually much faster than transport across the BBB and hence is particularly important for local penetration of a solute. The ECS consists of the hyaluronan-based ECM and a fluid phase, and is characterized by a volume fraction $\alpha\left(=V_{\mathrm{ECS}} / V_{\mathrm{brain}}\right)$ of 0.15-0.30 (Sykova and Nicholson, 2008). The fluid phase serves as a reservoir for extracellular ions necessary for generating action potentials, a medium for transporting molecules such as neurotransmitters involved in signaling, and for transporting essential molecules between microvessels and cells in the brain. The extracellular volume fluctuates during normal brain function and decreases during development and aging (Sykova and Nicholson, 2008; Kroeger et al., 2010).

The geometry of the extracellular space has been modeled as an interconnected network of sub-100 nm pores resembling sheets and tunnels (Sykova and Nicholson, 2008; Kinney et al., 2013). Sheets represent regions where the plasma membranes of two cells are in close proximity, similar to two parallel plates, and tunnels correspond to approximately cylindrical channels. The geometry of the network of pores in the ECS restricts diffusion in the brain 
compared to free diffusion, and is characterized by the tortuosity, $\lambda$ which is defined by the ratio $\left(\Delta_{\text {free }} / \Delta_{\text {brain }}\right)^{1 / 2}$ where $D_{\text {free }}$ is the diffusion coefficient in solution and $D_{\text {brain }}$ is the diffusion coefficient in the brain (Nicholson, 2001). The tortuosity takes into account the fact that molecules must detour around cells during transport.

Results from tracer experiments suggest a value of $\lambda \approx 1.6$ in the rat brain. Since the diffusion coefficient for $\mathrm{K}^{+}$and $\mathrm{Cl}^{-}$ ions in aqueous solution is around $2 \times 10^{-5} \mathrm{~cm}^{2} \mathrm{~s}^{-1}$, a tortuosity of 1.6 implies a diffusion coefficient in the brain of around $0.8 \times 10^{-5} \mathrm{~cm}^{2} \mathrm{~s}^{-1}$. Analysis of in vivo experiments using probes with different hydrodynamic radii suggests effective pore dimensions of about $40 \mathrm{~nm}$ between parallel plates and about $60 \mathrm{~nm}$ for cylindrical channels (Thorne et al., 2005; Thorne and Nicholson, 2006). As a result, the transport of molecules or particles approaching these dimensions will be limited due to steric hindrance and drag by the pore walls (Thorne et al., 2005; Thorne and Nicholson, 2006).

While global biophysical parameters such as ECS volume fraction, tortuosity, and effective pore size have been estimated, the details of the physico-chemical properties that control transport between capillaries and neurons and other cells in the brain remain to be established. Transport in the ECS may also be modulated by dead-end branches in the ECS network, transient binding with the ECM in the extracellular space, transient binding with cell membranes, or cell uptake (Sykova and Nicholson, 2008).

\section{SHEAR STRESS}

Blood pressure exerts a force normal to a vessel wall that imposes a circumferential stress on the vessel, whereas blood flow results in a frictional drag, or shear stress, parallel to the endothelium in the direction of blood flow. These stresses play an important role in regulating endothelial cell morphology and function, and in mediating a wide range of signaling and transport processes between the vascular system and surrounding tissue (Chien, 2007; Hahn and Schwartz, 2009; Johnson et al., 2011; Conway and Schwartz, 2012). These stresses are also thought to play an important role in regulation of the blood-brain barrier (KrizanacBengez et al., 2004; Neuwelt et al., 2008, 2011; Tarbell, 2010; Cucullo et al., 2011).

For an ideal Newtonian fluid (incompressible), the shear stress $\tau$ in a straight cylindrical vessel under constant laminar flow is given by the Poiseuille equation: $\tau=4 \mu \mathrm{Q} / \pi \mathrm{r}^{3}$ where $\mu$ is the dynamic viscosity, $Q$ is the volumetric flow rate, and $r$ is the radius of the lumen. Therefore, the magnitude of the shear stress on the endothelium is proportional to the flow rate and viscosity, and inversely proportional to $r^{3}$. Consequently, endothelial cells in vessels with high flow rate and small diameter are exposed to large shear stress.

The viscosity of blood is about $4 \mathrm{cP}(0.004 \mathrm{~Pa} \cdot \mathrm{s})$, significantly larger than the viscosity of water of $0.7 \mathrm{cP}(0.0007 \mathrm{~Pa} \cdot \mathrm{s})$ at $37^{\circ} \mathrm{C}$, primarily due to the presence of red blood cells. Typical time averaged values of shear stress are 4-30 dynes $\mathrm{cm}^{-2}$ in the arterial circulation and 1-4 dynes $\mathrm{cm}^{-2}$ in the venous circulation (Turitto, 1982; Kamiya et al., 1984; Papaioannou and Stefanadis, 2005; Koutsiaris et al., 2007; Dolan et al., 2013).
The flow rate in capillaries is typically from 6 to $12 \mathrm{~nL} \mathrm{~min} \mathrm{~m}^{-1}$ corresponding to a shear stress of 10-20 dynes $\mathrm{cm}^{-2}$ for a capillary $10 \mu \mathrm{m}$ in diameter (taking $\mu=1 \mathrm{cP}$ or $0.001 \mathrm{~Pa} \mathrm{~s}$; Kamiya et al., 1984).

As described above, the viscosity of bulk blood is around 4 $\mathrm{cP}$, significantly higher than the viscosity of water, due in large part to the density of red blood cells. A complication arises in small capillaries since cells tend to avoid the vessel walls resulting in a cell-free layer within about $3 \mu \mathrm{m}$ of the surface that has a viscosity close to that of water, a phenomenon known as the Fahraeus-Lindqvist effect (Fahraeus and Lindqvist, 1931). For large diameter vessels, this effect is negligible and the effective viscosity is close to the bulk viscosity of blood. However, for smaller diameter vessels, the cell-free layer can become a significant fraction of the cross-sectional area resulting in a decrease in effective viscosity.

\section{TRANSPORT ACROSS THE BBB INTRODUCTION}

The barrier function of the BBB is critical for regulating transport to the brain, but also represents a significant roadblock in delivering drugs to the brain (Pardridge, 2003, 2005, 2006, 2008, 2010; Hawkins and Davis, 2005; Ohtsuki and Terasaki, 2007). Only very few CNS disorders, such as depression, schizophrenia, chronic pain, and epilepsy, are currently treatable with small molecule drug therapy. The BBB is the major roadblock in developing therapies for CNS diseases including neurodegenerative diseases, cerebrovascular diseases, and brain cancer. Therefore, methods to study the transport of drugs and other molecules across the blood-brain barrier are key to understanding how the BBB regulates transport and will be invaluable for drug discovery and the treatment of CNS diseases (Cecchelli et al., 2007; Kuhnline Sloan et al., 2012).

The formation of tight junctions effectively eliminates paracellular transport across the blood-brain barrier (de Boer et al., 2003; Cecchelli et al., 2007; Abbott et al., 2010; Giacomini et al., 2010). Transcellular transport can occur through various mechanisms (Lee et al., 2001; de Boer et al., 2003; Cecchelli et al., 2007; Ohtsuki and Terasaki, 2007; Ueno, 2009; Abbott et al., 2010; Giacomini et al., 2010), as illustrated in Figure 3. Small lipophilic molecules can enter the brain via passive diffusion across the luminal and abluminal cell membranes. To regulate passive transport into the brain, efflux pumps return many unwanted molecules back to the circulatory system. Small polar molecules, such as glucose, amino acids, organic anions and cations, and nucleosides, can cross the blood-brain barrier by carrier-mediated transport. These solute carriers may be specific to one molecule or multispecific to several molecules. Large solutes, such as proteins and peptides, are transported across the $\mathrm{BBB}$ by receptor-mediated or adsorption-mediated endocytic transport. Highlighting the important role of transport, it has been estimated that $10-15 \%$ of all proteins in the neurovascular unit are transporters (Enerson and Drewes, 2006). As a result of this regulated transport, there can be large differences in the concentration of amino acids and proteins while differences the concentration of ions in the blood and cerebral spinal fluid are relatively small (Abbott et al., 2006). 


blood
membrane
transport

FIGURE 3 | Transport systems at the blood-brain barrier. (1) Small ions and water molecules can cross the blood-brain barrier through ion channels. (2) Small lipophilic molecules that are soluble in the hydrophobic core of the cell membrane can be transported passively across the cell. (3) Essential polar molecules that cannot diffuse through the cell membrane are shuttled across the cell membranes by carrier-mediated transport. These solute carriers may be directional, in or out of the cell, or bidirectional. Other molecules can be actively transported across endothelial cell membranes by carrier-mediated transporters, receptor-mediated transporters, adsorption-mediated transcytosis, or efflux pumps.
Passive transport is a way to bypass the array of substrate specific transport systems that are designed to regulate transport across the blood-brain barrier. In general, passive transcellular transport is limited to small molecules that have a combination of sufficient hydrophilicity to be soluble in water and sufficient lipophilicity to be soluble in the hydrophobic core of the lipid bilayer. Small gaseous molecules such as $\mathrm{O}_{2}$ and $\mathrm{CO}_{2}$ can diffuse through the cell membrane, as well as small molecules such as barbiturates, ethanol, and caffeine. Almost no large molecules and $98 \%$ of all small molecules do not cross the BBB.(Pardridge, $1998,2010)$ In general, molecules that passively diffuse across the BBB have a $M W<500 \mathrm{Da}, \log P_{\text {oct }}$ in the range 2-4, and the number of hydrogen bond donors is less than 5 (Avdeef, 2001; Lipinski et al., 2001). Many molecules that cross the membrane by passive transport are subsequently transported back to the vascular system by efflux pumps.

The details of transport from a capillary into the brain remain poorly understood. As described above, BMECs are surrounded by pericytes that extend processes over the capillary surface, a 50-100 nm thick basement membrane, and astrocyte end-feet that almost completely surround the outside of the capillaries.
Transport across an endothelial cell in a capillary may not be radially symmetric since the cell thickness, and hence diffusion length, is dependent on position. From transmission electron microscope images of rat brain capillaries, the endothelial cell thickness ranges from about $0.2 \mu \mathrm{m}$ away from the nucleus to about $0.9 \mu \mathrm{m}$ in the vicinity of the nucleus (Farkas and Luiten, 2001; Nicaise et al., 2009). Once transported across the endothelium, a molecule enters the basement membrane where it can be transported into a pericyte or astrocyte, or can diffuse laterally to a gap between astrocyte end-feet and into the extracellular space. To predict the spatial and temporal distribution of a molecule in the brain will require detailed characterization of the transport properties of the cellular and matrix components of the neurovascular unit, along with an understanding of how these properties change with time, for example during development, aging, and disease.

\section{LIPOPHILICITY}

Lipophilicity is the affinity of a molecule for a lipophilic environment (McNaught and Wilkinson, 1997). The partition coefficient $P$ is the ratio of the concentration of the molecule in a solvent 
such as octanol to the concentration in water $\left(P_{\text {oct }}\right.$; Avdeef, 2001; Waterhouse, 2003). The solvent is selected to mimic the hydrophobic environment of the core of a lipid bilayer. Explicitly, $P$ is defined for all neutral species and is $\mathrm{pH}$ independent. If the molecule can be ionized then the lipophilicity is determined by the distribution coefficient $\mathrm{D}$ which takes into account ionized species. Depending on the $\mathrm{pKa}$, the distribution coefficient will show a $\mathrm{pH}$-independent regime where the molecule is neutral and $\mathrm{a} \mathrm{pH}$ dependent regime where the molecule is ionized. In general, ionization results in increased solubility but decreased partitioning to octanol.

The lipid composition of the membrane of human BMECs is $\sim 33 \%$ phosphatidyl choline (PC), 25\% phosphatidyl ethanolamine (PE), 17\% sphyngomyelin (Sph), 11\% phosphatidyl serine (PS), 4.8\% phosphatidyl inositol (PI; Siakotos et al., 1969; Tewes and Galla, 2001).

\section{IN VITRO MEASUREMENTS \\ The transwell assay}

The development of an in vitro platform to study transport across the blood-brain barrier has proven challenging. This is not surprising, as brain capillary endothelial cells transduce signals from surrounding astrocytes, pericytes, and from the vascular system. In vitro transport measurements are usually performed using a transwell assay where a confluent monolayer of endothelial cells is formed on a porous support separating two chambers (Figure 4). The permeability can be determined from the transport of a drug or fluorescent probe from the donor side to the acceptor side. Alternatively, ion transport can be characterized in terms of the electrical impedance of the monolayer.

The transwell assay is widely used to study absorption of orally administered molecules in the intestine (Artursson, 1991; Artursson et al., 2001; Stenberg et al., 2001; Hubatsch et al., 2007). Caco- 2 cells of passage 30-45 are plated on polymer membranes with $1 \mu \mathrm{m}$ pores and cultured for 20-23 days to confluence. Confluence is confirmed by a resistance measurement of over 200 $\Omega \mathrm{cm}^{2}$. After confluence is reached, permeability measurements can be made across the monolayer. A permeability of $>10^{-6} \mathrm{~cm}$ $\mathrm{s}^{-1}$ is correlated with $100 \%$ oral absorption, whereas a permeability of $<10^{-7} \mathrm{~cm} \mathrm{~s}^{-1}$ is correlated with less than $1 \%$ oral absorption. Permeabilities for common drugs include $5.3 \times 10^{-5} \mathrm{~cm}$

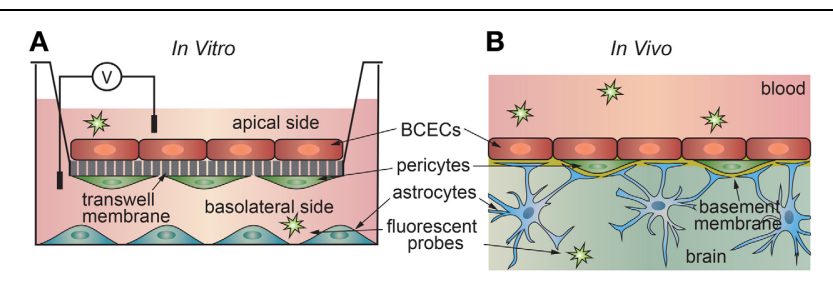

FIGURE 4 | Schematic illustration of (a) in vitro and (b) in vivo transport measurements. (A) In the 2D transwell assay, a monolayer of cells is formed on a porous membrane separating two compartments. Astrocytes and/or pericytes may be seed on the opposite side of the membrane or in the output chamber. (B) In vivo studies, a solute is injected into the blood of an animal model, and the penetration into the brain measured using a suitable chemical detection assay or imaging technique. $\mathrm{s}^{-1}$ for ibuprofen, $2.0 \times 10^{-6} \mathrm{~cm} \mathrm{~s}^{-1}$ for benzyl penicillins, and $1.6 \times 10^{-7} \mathrm{~cm} \mathrm{~s}^{-1}$ for doxorubicin (Yee, 1997). Caco-2 cells are also widely used for determining whether a substance is a P-gp substrate by measuring the bidirectional permeability (Balimane et al., 2006).

2D models of the blood-brain barrier for transport studies historically utilize monolayers of type II Madin-Darby canine kidney (MDR1-MDCK) cells, genetically engineered to express the Pg-p pump. Other cell types used for the transwell assay are primary BMECs isolated from human or animal brain tissue. These cells are usually plated on transwell membranes coated with rat tail collagen I or basement membrane proteins such as collagen IV, fibronectin, laminin (Tilling et al., 1998). BMECs are often co-cultured with astrocytes and/or pericytes to induce blood-brain barrier properties. Astrocytes are commonly cultured in the lower compartment of the transwell dish, either on the opposite side of the membrane from the endothelial cells or on the bottom of the dish to provide secreted factors (Siddharthan et al., 2007). Astrocyte-conditioned media is also commonly used in transwell systems, as the soluble factors secreted by astrocytes, such as bFGF and GDNF, have been shown to increase tight junction properties (Abbott et al., 2006). The influence of pericytes on transport in the transwell assay is not well-understood and may be dependent on their stage of differentiation (Thanabalasundaram et al., 2011). Tri-culture models with endothelial cells plated on a transwell membrane, either astrocytes or pericytes on the opposite side of the membrane, and the third cell type plated on the bottom of the dish have been found to improve blood-brain barrier properties compared to co-culture (Nakagawa et al., 2009; Hatherell et al., 2011).

\section{Transendothelial electrical resistance (TEER)}

The first in vivo transendothelial electrical resistance measurements were performed on frog brain microvessels in a twoelectrode configuration with one electrode inserted into the vessel and the other used to measure the voltage drop as a function of distance along the vessel (Crone and Olesen, 1982). The voltage drop along a cylindrical vessel can be related to the TEER:

$$
V(\mathrm{x})=V(0) \exp \left(-\frac{x}{\lambda}\right)
$$

where $V(x)$ is the voltage at distance $x$, and $\lambda$ is the characteristic length which depends on the vessel radius and the resistivity of blood. The transendothelial resistance $R_{m}$ of the endothelial cells defining the lumen of the vessel is then determined from:

$$
R_{m}=2 \pi a R_{i} \lambda
$$

where $R_{i}$ is the internal resistance of the capillary $\left(\Omega \mathrm{cm}^{-1}\right)$ and $a$ is the vessel diameter $(\mathrm{cm})$. The internal resistance is given by:

$$
R_{i}=\frac{\rho_{i}}{\pi a^{2}}
$$

where $\rho_{i}$ is the resistivity of blood $(\Omega \mathrm{cm})$. Blood resistivity is exponentially dependent on the hematocrit, with a typical value 
of around $120 \Omega \mathrm{cm}$ corresponding to a $40 \%$ hematocrit. Blood plasma has a resistivity of around $50 \Omega \mathrm{cm}$ (see Supplementary Information). Note that the resistance is normalized to unit area of the endothelium and has units of $\Omega \mathrm{cm}^{2}$.

From these experiments the average resistance was determined to be $1840 \Omega \mathrm{cm}^{2}$ (Crone and Olesen, 1982). Subsequent experiments with rat brain surface microvessels determined the average resistance of venous microvessels to be $800 \Omega \mathrm{cm}^{2}$, and the average resistance of arterial microvessels to be $2000 \Omega \mathrm{cm}^{2}$, with an overall average of $1500 \Omega \mathrm{cm}^{2}$ (Butt and Jones, 1992). TEER measurements have been widely used to characterize tight junctions (Madara, 1998; Franke et al., 1999; Gumbleton and Audus, 2001; Reichel et al., 2003; Deli et al., 2005; Shen et al., 2011).

TEER measurements using the transwell assay generally result in resistances much lower than the values of 1500-2000 $\Omega \mathrm{cm}^{2}$ reported for in vivo measurements. Values in excess of $150-200 \Omega \mathrm{cm}^{2}$ are generally considered suitable for studying solute and drug transport (Reichel et al., 2003). There are two contributing factors to this difference. First, the transwell assay does not recapitulate all of the physical and biological features of the $\mathrm{BBB}$, resulting in the formation of cell-cell junctions that are not quite as effective in restricting paracellular transport. Second, there is usually a short circuit path due to incomplete monolayer formation or poor adhesion to the walls of the transwell support. In the transwell assay, if we consider a short circuit resistance in parallel with the endothelial resistance then the measured resistance is given by:

$$
R_{\text {meas }}=R_{S}+\frac{1}{\left(\frac{R_{S C}}{1-f_{c}}\right)^{-1}+\left(\frac{R_{m}}{f_{c}}\right)^{-1}}
$$

where $R_{s}$ is the solution resistance, $R_{\mathrm{sc}}$ is the short circuit resistance, $R_{m}$ is the endothelial cell resistance, and $f_{c}$ is the fraction of the transwell surface covered with a confluent monolayer of endothelial cells. Note that when $f_{c} \rightarrow 1, R_{\text {meas }} \approx R_{m}$ as long as $R_{m} \gg R_{s}$. Taking $R_{s}=2 \Omega \mathrm{cm}^{2}, R_{\mathrm{sc}}=5 \Omega \mathrm{cm}^{2}$, and $R_{m}=$ $1500 \Omega \mathrm{cm}^{2}$, the typical range of resistances for in vitro measurements correspond to a coverage fraction from 0.90 to 0.98 . The problem associated with a short circuit path at the perimeter of the TEER device can be overcome by plating cells on microfabricated electrodes. The disadvantage of using microfabricated electrodes is that there is no solution reservoir underneath the cell monolayer making it very difficult to perform transport measurements.

While the relatively low TEER values usually obtained in transwell experiments $\left(150-200 \Omega \mathrm{cm}^{2}\right)$ makes it difficult to compare experiments quantitatively to in vivo conditions, it still allows qualitative assessment of conditions that induce BBB properties. For example, the important role of astrocytes in inducing $\mathrm{BBB}$ properties is demonstrated by the increase in TEER values observed by co-culture of BCECs with astrocytes or astrocyte-conditioned media (debault and Cancilla, 1980; Abbott, 2002). Similarly, the increase in TEER observed in tri-culture models provides additional evidence for the role of pericytes in the induction of $\mathrm{BBB}$ properties.

\section{PERMEABILITY}

The rate of transport of a solute across a barrier is characterized by the permeability, which is defined as the flux through unit area under unit concentration gradient and has units of $\mathrm{cm} \mathrm{s}^{-1}$. It is implicitly assumed that there is no hydrostatic or osmotic pressure difference across the barrier (Kedem and Katchalsky, 1958). In the analysis of transport across the blood-brain barrier, the endothelium is generally considered a black box with first order rate constants $k_{\text {in }}$ and $k_{\text {out }}$ where $k_{\text {in }}$ describes transport of a solute into the brain and $k_{\text {out }}$ describes the reverse process (see Figure 5).

The flux (moles $s^{-1}$ ) of a solute across a volume element is given by:

$$
J=\frac{d N}{d t}=k_{\text {in }} c_{\text {in }}-k_{\text {out }} c_{\mathrm{out}}
$$

where $N$ is the number of moles transported across the volume element, $c_{\text {in }}$ is the concentration $\left(\mathrm{mol} \mathrm{cm}{ }^{-3}\right)$ on the input side

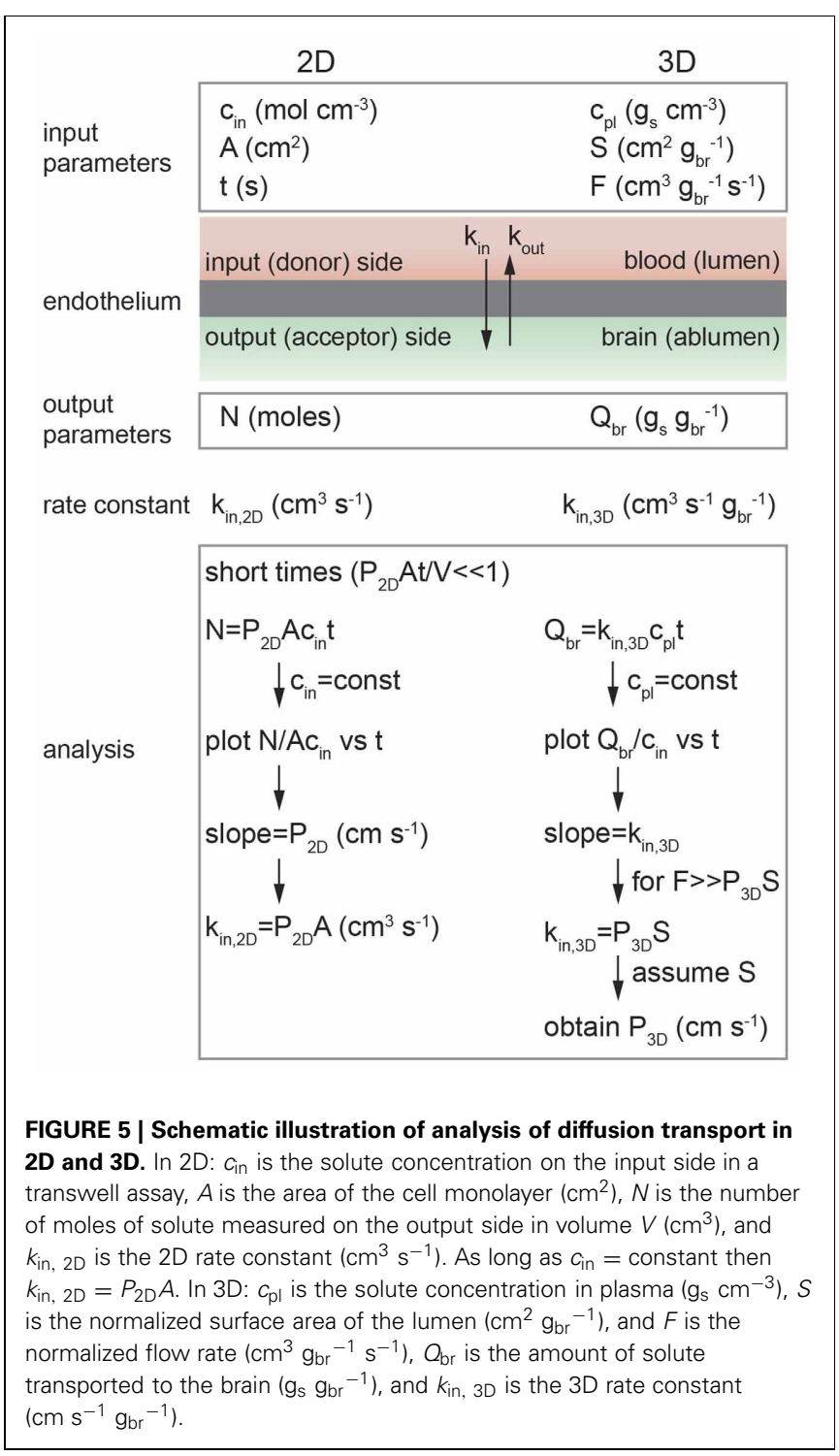




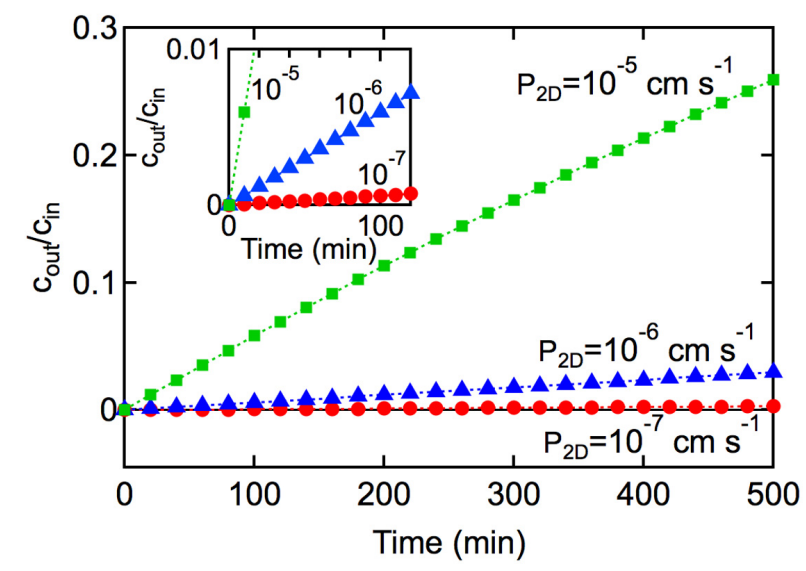

FIGURE 6 | Kinetics of solute transport across a 2D monolayer. $c_{\text {out }} / c_{\text {in }}$ is plotted as a function of time $t$, with $A=1 \mathrm{~cm}^{2}$, and $V=1 \mathrm{~cm}^{3}$ for ( $\square$ ) $P_{2 \mathrm{D}}=10^{-5} \mathrm{~cm} \mathrm{~s}^{-1}$, and $(\boldsymbol{\Delta}) 10^{-6} \mathrm{~cm} \mathrm{~s}^{-1}$, and $(\bullet) P_{2 D}=10^{-7} \mathrm{~cm} \mathrm{~s}^{-1}$, At short times (inset) where $P_{2 \mathrm{D}} A t / V \ll 1$, the slope is $P_{2 \mathrm{D}} A / V$ and the rate constant can be obtained from $k_{\text {in, } 2 \mathrm{D}}=P_{2 \mathrm{D}} A$.

and $c_{\text {out }}$ is the concentration on the output side. In all experiments it is implicitly assumed that paracellular transport across the tight junctions is negligible. For the case where transport is dominated by passive diffusion across the cell membranes then $k_{\text {in }}=k_{\text {out }}$ assuming negligible difference in lipid composition between the luminal and abluminal membranes. However, if a solute is a substrate for a transporter, such as an efflux pump then $k_{\text {in }} \neq k_{\text {out }}$.

The flux may be normalized in different ways, depending on the type of experiment. For example, the in vitro transwell assay is a 2D assay where the input and output compartments are separated by a monolayer of endothelial cells. In contrast, in vivo brain perfusion is a $3 \mathrm{D}$ assay. The difficulties in performing in vivo transport measurements can make comparison of $2 \mathrm{D}$ and $3 \mathrm{D}$ measurements somewhat confusing, for example, the in vitro (2D) rate constant $k_{\text {in, } 2 \mathrm{D}}$ is normalized to unit area whereas the in vivo (3D) rate constant $k_{\mathrm{in}, 3 \mathrm{D}}$ is usually normalized to unit mass.

\section{D TRANSPORT}

In vitro transport studies are typically performed using a $2 \mathrm{D}$ transwell assay where a confluent monolayer of endothelial cells on a porous support is located between input and output chambers (Figure 4) (Siflinger-Birnboim et al., 1987; Karlsson and Artursson, 1991; Artursson, 1991; Adson et al., 1994; Cecchelli et al., 1999; Youdim et al., 2003; Deli et al., 2005). A solute, typically radio-labeled or fluorescently-labeled, is introduced into the input chamber and the amount accumulated in the output chamber is measured as a function of time, typically over a period of 1-2 h (Bowman et al., 1983; Audus and Borchardt, 1986; Shah et al., 1989; Karlsson and Artursson, 1991; Artursson, 1991; Freed et al., 2001; Chappa et al., 2006; Summerfield et al., 2007). The concentration of solute on the input side $c_{\text {in }}\left(\mathrm{mol} \mathrm{cm}{ }^{-3}\right)$ and the area $A\left(\mathrm{~cm}^{2}\right)$ of the monolayer are the input parameters, and the concentration of solute on the output side is measured as a function of time. Note that $c_{\text {out }}=N / V$ where $N$ is the number of moles of solute and $V\left(\mathrm{~cm}^{3}\right)$ is the fluid volume in the output chamber.

Integrating Fick's first law and recognizing that $k_{\mathrm{in}, 2 \mathrm{D}}=P_{2 \mathrm{D}} A$ where $P_{2 \mathrm{D}}$ is the permeability $\left(\mathrm{cm} \mathrm{s}^{-1}\right)$, we obtain:

$$
N(t)=V c_{\text {in }}\left(1-\exp \left(-\frac{P_{2 \mathrm{D}} A}{V} t\right)\right)
$$

(see Figure 6 and Supplementary Information) (Kedem and Katchalsky, 1958; Siflinger-Birnboim et al., 1987; Dawson, 1991; Tran et al., 2004)At short times where $P_{2 \mathrm{D}} A t \ll V$, the exponential term can be linearized and hence:

$$
N(t)=P_{2 \mathrm{D}} A c_{\mathrm{in}} t
$$

In the derivation of Equation 7 it is assumed that: (1) the concentration of solute in the input chamber $\left(c_{\mathrm{in}}\right)$ is approximately constant, (2) transport from the output chamber to the input chamber can be neglected $\left(k_{\text {out }} \mathcal{c}_{\text {out }} \rightarrow 0\right)$, and (3) transport is dominated by passive diffusion across the cell membrane (see Supplementary Information). Experimentally, the permeability can be obtained from the slope of a plot of $N(t) / A c_{\text {in }}$ vs. time at short times where $P_{2 \mathrm{D}} A t / V \ll 1$. Alternatively, $c_{\text {out }} / c_{\text {in }}$ can be plotted against $t$ where the slope is $P_{2 \mathrm{D}} A / V$. The rate constant can then be obtained from $k_{\text {in, } 2 \mathrm{D}}=P_{2 \mathrm{D}} A$.

Experimentally, transport of a solute from the input chamber to the output chamber involves transport across the aqueous boundary layer above the cell monolayer, transport across the cell monolayer, and transport through the porous membrane. These steps are in series and hence we can write:

$$
\frac{1}{P_{2 \mathrm{D}}}=\frac{1}{P_{m}}+\frac{1}{P_{f}}+\frac{1}{P_{S}}
$$

where $P_{m}$ is the permeability coefficient of the cell monolayer, $P_{f}$ is the permeability coefficient of the transwell membrane, and $P_{S}$ is the permeability of the boundary layer above the cell monolayer (Barry and Diamond, 1984; Karlsson and Artursson, 1991; Artursson, 1991; Adson et al., 1994; Avdeef et al., 2005). To ensure that the measured permeability coefficient $P_{2 \mathrm{D}}=P_{m}$ requires that $P_{m} \ll P_{f}$ and $P_{S}$. The influence of $P_{f}$ and $P_{S}$ can be approximated using diffusion models (Karlsson and Artursson, 1991 and Artursson, 1991; Avdeef et al., 2005), or reduced by stirring (decreasing 1/P ; Cecchelli et al., 1999; Youdim et al., 2003; Summerfield et al., 2007) and using a transwell filter with larger pores (decreasing $1 / P_{f}$; Siflinger-Birnboim et al., 1987; Adson et al., 1994). Alternatively, the sum of $1 / P_{f}$ and $1 / P_{S}$ can be measured in a control experiment with no cells on the transwell membrane.

Permeability coefficients obtained using the transwell assay are typically in the range from $10^{-7}$ to $10^{-3} \mathrm{~cm} \mathrm{~s}^{-1}$ (Pardridge et al., 1990; Deli et al., 2005; Summerfield et al., 2006, 2007), somewhat higher than values measured in vivo. Typical $P_{2 \mathrm{D}}$ values for marketed CNS drugs vary between $10^{-7}$ and $10^{-5} \mathrm{~cm} \mathrm{~s}^{-1}$ (Summerfield et al., 2007). The measured permeability coefficient $P_{2 \mathrm{D}}$ increases with lipophilicity reaching a plateau around 
$\log P_{\text {oct }}=3$. Increasing the lipophilicity above $\log \mathrm{P}_{\text {oct }}=3$ results in a reduction of $P_{2 D}$ (Summerfield et al., 2007).

In general, $P_{2 \mathrm{D}}$ increases approximately linearly with increasing lipophilicity. Deviations from this trend are generally due to violation of the assumption that transport is dominated by passive diffusion across the cell membrane. Apparent increases in $P_{2 D}$ can result from active transport and apparent decreases may be due to the influence of efflux pumps. At higher lipophilicities, solute trapping in the cell membrane and internal vesicles can lead to a lower apparent solute concentration in the output chamber and hence a lower apparent permeability.

\section{RESECTED VESSEL ASSAY}

As described above, the in vitro transwell assay is widely used to study passive transport across BMECs. However, the transwell assay has limited utility in studying active transport, since it is difficult to recapitulate the physiological polarization of pumps and transporters. A complementary method that is particularly useful in studying efflux pumps is the resected vessel assay (Schramm et al., 1995; Miller, 2003; Hartz et al., 2004; Bauer et al., 2005; Wang et al., 2010; Campos et al., 2012). In this assay, a resected brain capillary, typically from a rat or mouse brain, is transferred to a dish and immersed in buffer. A fluorescently-labeled solute of interest is then introduced into the media and uptake is measured by recording the fluorescence in the lumen of the vessel. The solute is usually a substrate for a particular transporter. For example, NBD-CSA is a fluorescent derivative of cyclosporine $A$ that is a substrate for the P-gp pump (Didier et al., 1996), BODIPYFL prazosin is a fluorescently labeled substrate for BCRP (Robey et al., 2001), and Texas red is a fluorescent substrate for MRP2 (Bauer et al., 2008).

In the resected vessel assay, the solute is introduced into the bath, corresponding to the brain side of the vessel. The solute is transported across the abluminal membrane, diffuses across the cell, and then is transported across the luminal membrane. In general, the solute concentration in the lumen increases with time and reaches a steady state value after 30-60 min. Common efflux pumps such as P-gp and BCRP1 are expressed preferentially on the luminal membrane, therefore the concentration of the solute in the lumen of the vessel can be larger than in the bath. Since the direction of transport is from the brain parenchyma side to the vessel lumen, inhibition of efflux pumps is expected to decrease the solute concentration in the vessel.

Transport across the endothelium of a resected vessel can be analyzed using the model in Figure 7A (Ye and Searson, unpublished). Assuming that intracellular transport is fast in comparison to passive transport across the membrane that the forward and backward rate constants for passive diffusion are the same $\left(k_{m}=k_{-m}\right)$, and that the solute concentration inside the cell is approximately constant, we obtain (see Supplementary Information):

$$
c_{\text {lum }}(t)=c_{\text {bath }}\left(1+\frac{k_{\text {pgp }}}{k_{m}}\right)\left(1-\exp \left(-\frac{t}{\tau}\right)\right)
$$

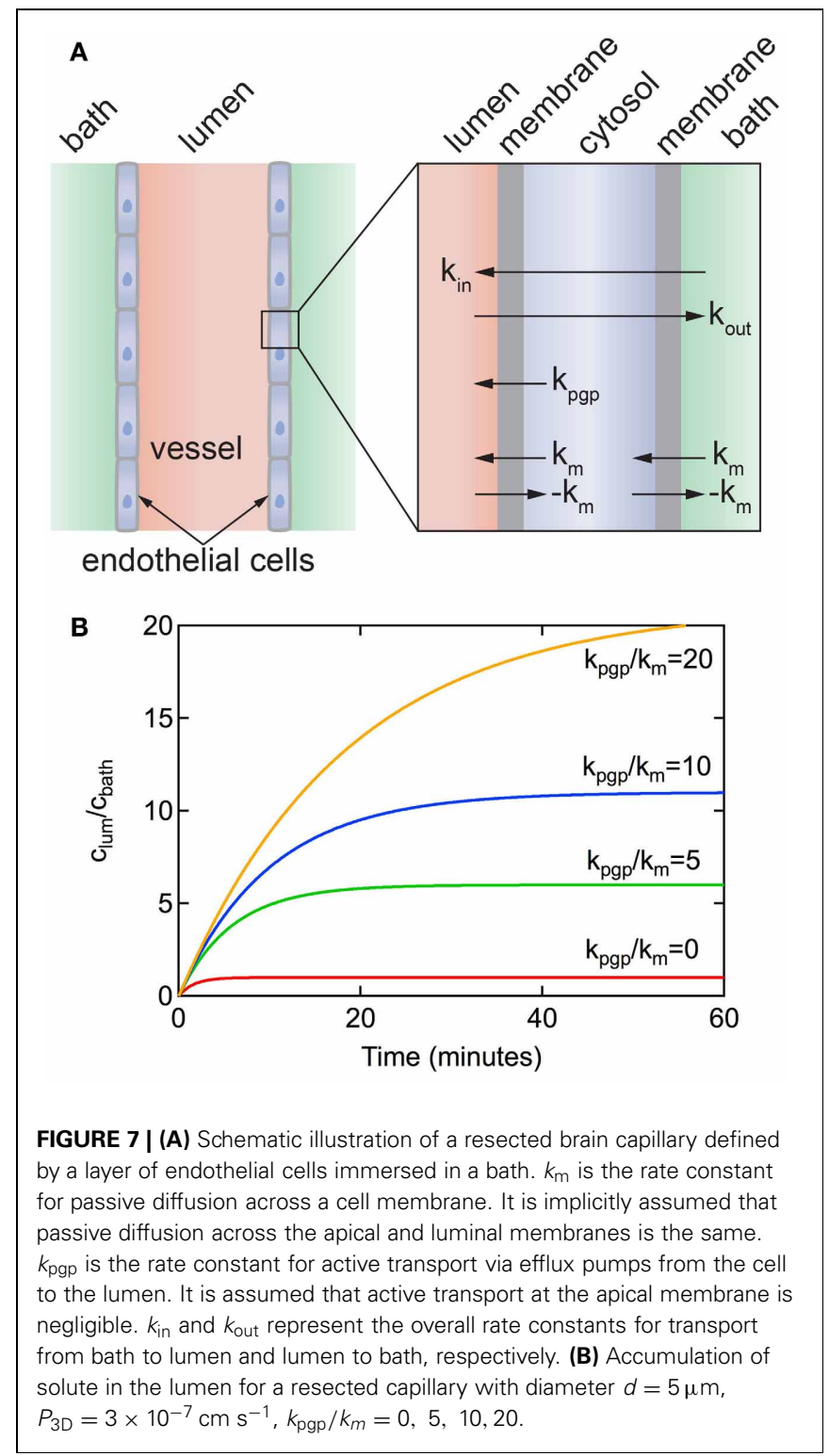

where the time constant $\tau$ is given by:

$$
\tau=\frac{r}{2 k_{m}}\left(2+\frac{k_{\text {pgp }}}{k_{m}}\right)
$$

Figure 7B shows plots of $c_{\text {lum }}(t) / c_{\text {bath }}$ vs. time for different values of $k_{\text {pgp }} / k_{m}$ for a vessel obtained from Equation 9. The concentration increases exponentially with time up to a steady-state value that is dependent on the ratio of $k_{\mathrm{pgp}} / k_{m}$. Note that without the P-gp transporter (i.e., $\left.k_{\text {pgp }}=0\right), c_{\text {lum }}(\infty) / c_{\text {bath }}=1$ and $k_{\text {in }}=k_{\text {out }}=k_{m} / 2$. As long as $k_{\text {pgp }} / k_{m}>0$ then $c_{\text {lum }}(\infty) / c_{\text {bath }}>$ 1. The time to reach a steady state solute concentration in the lumen increases with increasing $k_{\text {pgp }} / k_{m}$. Results from experiments reported in the literature show a time to reach steady state of 30-60 min (Hartz et al., 2004; Bauer et al., 2007; Hawkins et al., 2010; Cannon et al., 2012), consistent with $k_{\mathrm{pgp}} / k_{m} \approx 5$. 
From examination of the model it can be seen that rate constant $k_{m}=2 P_{0}$, where $P_{0}$ is the permeability for passive diffusion across the cell. $P_{0}$ can be equated to values obtained from in vitro transwell experiments or in vivo perfusion experiments where transport is dominated by passive diffusion. Typical values for $P_{0}$ range from $10^{-8}$ to $10^{-4} \mathrm{~cm} \mathrm{~s}^{-1}$ (Summerfield et al., 2007).

\section{D TRANSPORT}

Various in vivo techniques, such as intravenous injection, in situ brain perfusion, microdialysis, and positron emission tomography (PET), have been employed to determine the kinetics of drug transport across the BBB (Takasato et al., 1984; Ungerstedt, 1991; Pike, 2009; Kuhnline Sloan et al., 2012). Brain perfusion in rats is the most widely used technique for obtaining in vivo permeability values for small molecules and drugs (Hammarlund-Udenaes et al., 2009). Brain perfusion allows injection of a solute into the brain vasculature at higher flow rates and solute concentrations than can be achieved through systemic circulation and hence allows a wider range of solute permeabilities to be measured at a fixed perfusate concentration (Takasato et al., 1984; Hammarlund-Udenaes et al., 2009). Direct injection of the solute into the brain minimizes metabolic loss and plasma protein binding (Takasato et al., 1984). In this technique, the main blood supply leading to an animal's brain, often the common carotid artery (either left or right), is cannulated and connected to a perfusion system. Immediately after the animal's heart is stopped, a molecule of interest dissolved in physiological perfusate solution is infused into its brain typically for 5-300 s. Subsequently, the brain is removed and the ipsilateral hemisphere is dissected, weighed, and the solute concentration determined by LC-MS, HPLC, GC, or scintillation counting if the solute is radiolabeled (Smith, 2003).

The rate constant for in vivo transport $\left(k_{\text {in, 3D }}\right)$ can be obtained from the measured solute concentration in the brain $Q_{b r}\left(g_{s}\right.$ $\mathrm{gbr}^{-1}$; see Figure 5):

$$
Q_{b r}=k_{\mathrm{in}, 3 \mathrm{D}} c_{p 1} t
$$

where $c_{\mathrm{pl}}$ is the solute concentration in plasma $\left(\mathrm{g}_{\mathrm{s}} \mathrm{cm}^{-3}\right)$. In the derivation of Equation 11 it is assumed that: (1) the concentration of solute in plasma $\left(c_{\mathrm{pl}}\right)$ is constant and (2) the flux of solute out of the brain is not significant over the short infusion period (i.e., $\mathrm{k}_{\mathrm{out}} 3 \mathrm{D} \mathrm{c}_{\mathrm{br}} \approx 0$ ), which implies unidirectional transport (see Supplemental Information).

The rate constant, $k_{\mathrm{in}, 3 \mathrm{D}}$, is obtained from a single measurement of $Q_{\mathrm{br}} / c_{\mathrm{pl}}$ at a fixed infusion time $t$. The assumption of unidirectional transport can be confirmed by performing multiple perfusion experiments as a function of infusion time and determining the slope $\left(k_{\mathrm{in}, 3 \mathrm{D}}\right)$ of a linear regression to a plot of $Q_{\mathrm{br}} / c_{\mathrm{pl}}$ vs. time (Pathak et al., 2011).

While $k_{\text {in, 3D }}$ can be used to compare the in vivo transport kinetics of different solutes (Youdim et al., 2003), it cannot be compared directly to in vitro measurements $k_{\mathrm{in}, 2 \mathrm{D}}$. The rate constant $k_{\mathrm{in}, 3 \mathrm{D}}$ is related to the permeability $P_{3 \mathrm{D}}$ through the Crone-Renkin equation (see Supplemental Information) (Renkin, 1959; Crone, 1963):

$$
k_{\text {in, } 3 \mathrm{D}}=F\left(1-\exp \left(\frac{P_{3 \mathrm{D}} S}{F}\right)\right)
$$

where $F$ is the normalized flow rate $\left(\mathrm{cm}^{3} \mathrm{~s}^{-1} \mathrm{gbr}^{-1}\right)$ and $S$ is the normalized luminal surface area of vessels $\left(\mathrm{cm}^{2} \mathrm{gbr}^{-1}\right)$ in the brain. For the case where the flow rate $F \gg P_{3 \mathrm{D}} S$, which is equivalent to the initial assumption that the plasma concentration of the solute $c_{\mathrm{pl}}$ is constant, the exponential term can be linearized and hence:

$$
k_{\text {in, } 3 \mathrm{D}}=P_{3 \mathrm{D}} S
$$

Experimentally, as long as $F \geq 5 P_{3 \mathrm{D}} S$, then the error in measurement of $P_{3 \mathrm{D}}$ using Equation 13 is $\leq 10 \%$ (Smith and Takasato, 1986; Smith and Allen, 2003). S is taken to be $100-240 \mathrm{~cm}^{2}$ $\mathrm{g}_{\mathrm{br}}{ }^{-1}$, as determined by morphometric analysis of rat brain tissue sections (Gross et al., 1986; Fenstermacher et al., 1988).

Typical values of $P_{3 \mathrm{D}}$ for vascular tracers, nutrients, and drug molecules vary over 4 orders of magnitude from $10^{-8}$ to $10^{-4} \mathrm{~cm} \mathrm{~s}^{-1}$ (Takasato et al., 1984; Liu et al., 2004; Youdim et al., 2004; Summerfield et al., 2007). For small hydrophilic molecules, such as mannitol and sucrose, $P_{3 \mathrm{D}}$ is typically in the range $10^{-8}-10^{-7} \mathrm{~cm} \mathrm{~s}^{-1}$. In contrast, top selling antipsychotics and antidepressants such as venlafaxine, risperidone, buproprion, are generally small lipophilic molecules with $P_{3 \mathrm{D}}$ values between $10^{-5}$ and $10^{-4} \mathrm{~cm} \mathrm{~s}^{-1}$ (Summerfield et al., 2007). Caffeine has an intermediate lipophilicity $\left(\log P_{\mathrm{oct}}=-0.08\right)$ but relatively high permeability $\left(P_{3 \mathrm{D}}=4.2 \times 10^{-5} \mathrm{~cm} \mathrm{~s}^{-1}\right.$; Liu et al., 2004). Similarly, ethanol has an intermediate lipophilicity $\left(\log P_{\text {oct }}=\right.$ $-0.3)$ but a high permeability $\left(P_{3 \mathrm{D}}=1.1 \times 10^{-4} \mathrm{~cm} \mathrm{~s}^{-1}\right.$; Ohno et al., 1978; Takasato et al., 1984; Gratton et al., 1997).

The in vivo $3 \mathrm{D}$ permeability for many small molecules increases linearly with lipophilicity up to $\log P_{\text {oct }} \approx 3$, implying that transport from the blood to the brain is dominated by passive transport across the cell membranes (see Figure 8A) (Ohno et al., 1978; Rapoport et al., 1979; Smith and Takasato, 1986; Lipinski et al., 2001; Liu et al., 2004; Summerfield et al., 2007; Zhao et al., 2009). Deviations from this behavior are indicative of other transport mechanisms (Lipinski et al., 2001). For example, D-glucose has a very low lipid solubility $\left(\log P_{\text {oct }} \approx-3\right)$, but exhibits a high permeability coefficient $\left(P_{3 \mathrm{D}} \approx 10^{-5} \mathrm{~cm} \mathrm{~s}^{-1}\right)$ since transport is facilitated by the GLUT-1 transporter. Conversely, colchicine has relatively high lipid solubility $\left(\log P_{\text {oct }} \approx 2\right)$ but has a low permeability coefficient $\left(P_{3 \mathrm{D}} \approx 10^{-6} \mathrm{~cm} \mathrm{~s}^{-1}\right)$, since it is a substrate of the P-gp efflux pump (Youdim et al., 2003; Liu et al., 2004). Morphine has a relatively low permeability $\left(P_{3 \mathrm{D}}=1.1 \times 10^{-6}\right.$ $\mathrm{cm} \mathrm{s}^{-1}$ ) by drug standards, in part because it is a substrate for the P-gp pump (King et al., 2001), but highlights the fact that relevant doses can be achieved over reasonable time scales (Bouw et al., 2000; Tunblad et al., 2003; Hammarlund-Udenaes et al., 2008). Codeine (methyl morphine) has an $-\mathrm{OH}$ group on morphine substituted by a $-\mathrm{O}-\mathrm{CH}_{3}$ group, resulting in an increase in $\log P_{\text {oct }}$ from 0.2 to 1.24 , and increased permeability (Bostrom et al., 2008; Hammarlund-Udenaes et al., 2008). Dopamine has an intermediate lipophilicity $\left(\log P_{\text {oct }}=0.84\right)$ but low permeability $\left(P_{3 D}=1.1 \times 10^{-6} \mathrm{~cm} \mathrm{~s}^{-1}\right)$. However, L-dopa, a precursor that is metabolized to dopamine in the brain, has a very low lipophilicity $\left(\log P_{\text {oct }}=-2.53\right)$ but high permeability $\left(P_{3 \mathrm{D}}=6.6 \times 10^{-6} \mathrm{~cm}\right.$ 
$\mathrm{s}^{-1}$ ) since it is a substrate for the LAT-1 transporter (Gratton et al., 1997).

For $\log P_{\text {oct }} \geq 3$, both the apparent in vitro and in vivo permeabilities reach a maximum at about $10^{-4} \mathrm{~cm} \mathrm{~s}^{-1}$ (see Figure 8B) (Lipinski et al., 2001; Summerfield et al., 2007; Zhao et al., 2009). There are several factors that contribute to this apparent maximum. For solutes with high lipophilicity, transport becomes flowlimited. For in situ brain perfusion, the plasma concentration of the solute $c_{\mathrm{pl}}$ remains constant as long as $F \gg P_{3 \mathrm{D}} S$ (or $P_{3 \mathrm{D}} \ll$ $F / S$ ). Since the maximum flow rate is typically around $0.2 \mathrm{~cm}^{3}$ $\mathrm{s}^{-1} \mathrm{~g}_{\mathrm{br}}{ }^{-1}$, and assuming a luminal surface area of $100-200 \mathrm{~cm}^{2}$ $\mathrm{g}_{\mathrm{br}}{ }^{-1}$, the plasma concentration $c_{\mathrm{pl}}$ is expected to remain constant for values of permeability up to $P_{3 \mathrm{D}} \approx 10^{-4} \mathrm{~cm} \mathrm{~s}^{-1}$. At this limit and above, most of the injected solute is absorbed into the brain, and as a result, the in vivo permeability does not increase with lipophilicity for $\log P_{\text {oct }}>3$. For in vitro measurements, at high
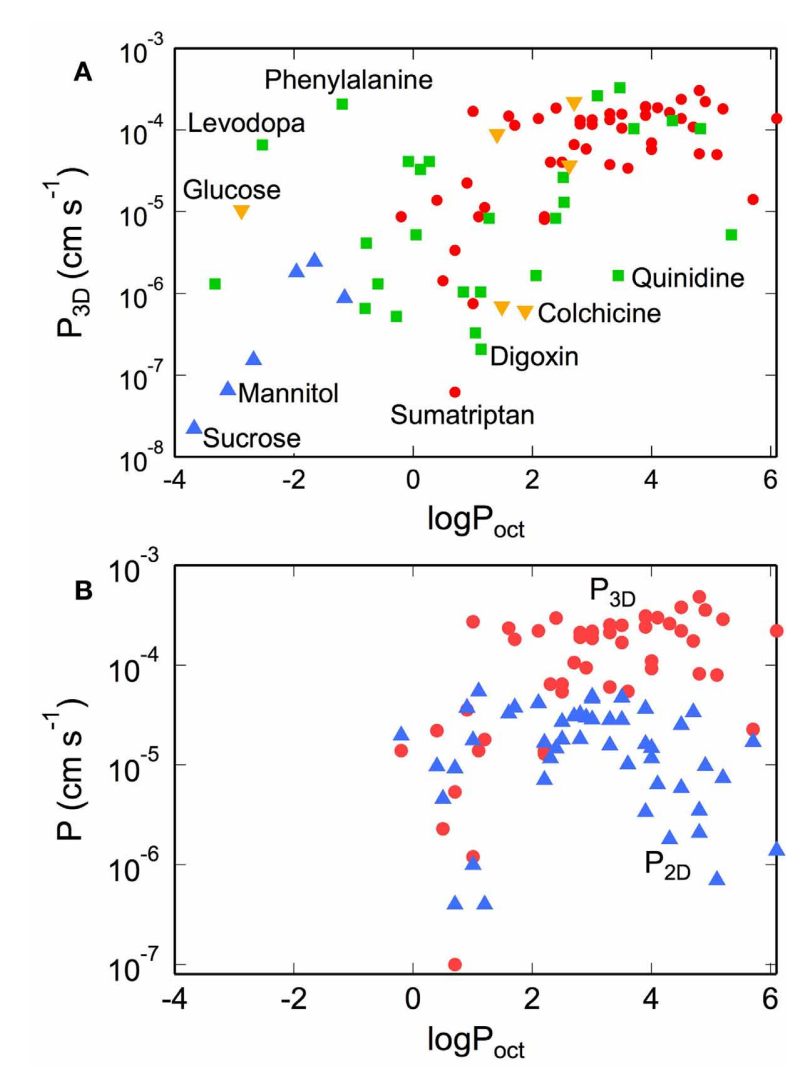

FIGURE 8 | (A) Permeability of tracers, nutrients, and drugs obtained from in situ rat brain perfusion vs. lipophilicity. (•) Summerfield et al. (2007), (A)

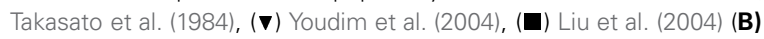
Comparison of permeability of various CNS drugs obtained from transwell assays on monolayers of MDR1-MDCK $\left(P_{2 D}\right)$ and in situ rat brain perfusions $\left(P_{3 D}\right) . P_{3 D}$ values were obtained from in situ rat brain perfusion measurements reported in the literature. For data reported as the permeability surface area products $\left(P_{3 D} S, \mathrm{~cm}^{3} \mathrm{~s}^{-1} \mathrm{gbr}^{-1}\right)$ we take $S=150 \mathrm{~cm}^{2} \mathrm{gbr}^{-1}$. Values of $P_{3 \mathrm{D}}$ where $S \neq 150 \mathrm{~cm}^{2} \mathrm{gbr}^{-1}$ were recalculated with $S=150 \mathrm{~cm}^{2} \mathrm{gbr}^{-1}$. Corresponding literature values for $\log P_{\text {oct }}$ were obtained from calculation Liu et al. (2004), Summerfield et al. (2007), and Youdim et al. (2004) or direct measurement of solute partitioning into octanol and water phases [Takasato et al. (1984)]. rates of uptake, the apparent permeability can become limited by transport across the boundary layer or the porous membrane, as described above, resulting in an apparent maximum in permeability.

A noticeable difference between $P_{2 \mathrm{D}}$ and $P_{3 \mathrm{D}}$ occurs at $\log P_{\mathrm{oct}}>3$. While $P_{3 \mathrm{D}}$ maintains a plateau in this regime, $P_{2 \mathrm{D}}$ decreases with increasing $\log P_{\text {oct }}$ for both MDCK and Caco2 models (Wils et al., 1994; Sawada et al., 1999; Summerfield et al., 2007). This effect can be explained by solute binding and absorption in the cell membrane (Kubinyi, 1977). As a result, fewer molecules are able to efflux from the endothelium into the output chamber, thus contributing to a decrease in the apparent permeability $\left(P_{2 D}\right)$. In the transwell assay, the amount of solute bound to the cell membrane, termed membrane retention or association, can be substantial (Sawada et al., 1999; Avdeef, 2001, 2003; Youdim et al., 2003; Fujikawa et al., 2007).

For in vivo transport, solute binding or trapping can be more complicated (Figure 9). Solute that is transported across the brain endothelium can diffuse through the interstitial fluid in the ECM, as described previously, and ultimately be taken up by neurons or glial cells in the brain. However, solute in the interstitial fluid can also bind to the ECM and hence be unavailable therapeutically. Characterization of in vivo transport therefore requires knowledge about additional parameters. In one approach, the dynamics of solute transport in vivo can be captured by three parameters: $K_{p, u}, P_{3 \mathrm{D}}$, and $V_{u, \text { brain }}$ (Hammarlund-Udenaes et al., 2008). $K_{p, u}$ is the ratio of unbound drug in the brain and blood at steady state, and typically has values between $0.02-3 . K_{p, u}=1$ for passive transport, $K_{p, u}<1$ for active efflux, and $K_{p, u}>1$ for active influx. $P_{3 \mathrm{D}}$ describes the permeability of transport into the brain and can vary by four orders of magnitude (Figure 8). For passive transport, $P_{3 \mathrm{D}}$ is expected to be related to the lipophilicity. The product $P_{3 \mathrm{D}} S\left(\mathrm{~cm}^{3} \mathrm{~s}^{-1} \mathrm{~g}_{\mathrm{br}}{ }^{-1}\right)$ corresponds to the net influx or clearance into the brain. $V_{u \text {, brain }}\left(\mathrm{mL} \mathrm{gbr}^{-1}\right)$ is a measure of the

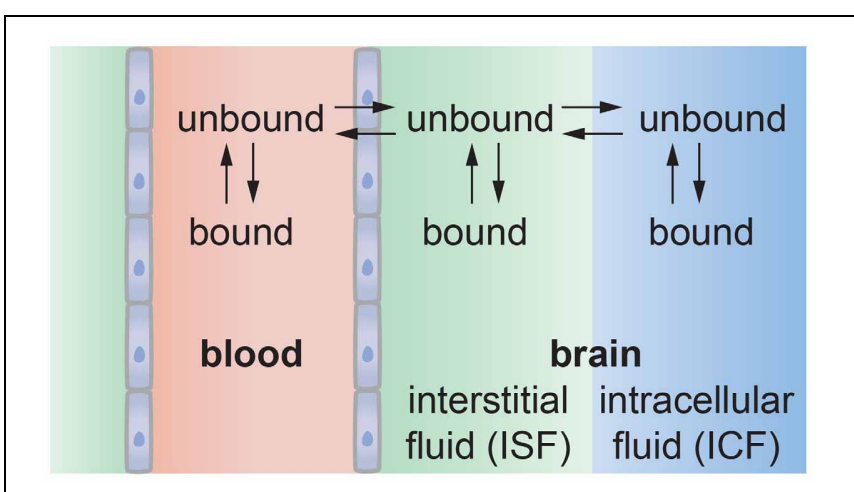

FIGURE 9 | Schematic illustration of solute transport from the vascular system into the brain. The solute may bind with proteins or other components in blood that may reduce the amount that can enter the brain. Solute transported across the endothelium may be partitioned between the interstitial fluid and intracellular fluid in neurons and glial cells. Solute in the interstitial fluid may be bound to the ECM, reducing the amount available for uptake by cells. 
distribution of the solute in the brain and is given by the ratio of the total amount of solute in the brain $\left(\mathrm{mol} \mathrm{gbr}^{-1}\right)$ to the unbound concentration in the interstitial fluid $\left(\mathrm{mol} \mathrm{mL}^{-1}\right)$. If all of the solute is in the interstitial fluid and there is no solute in the brain cells, then $V_{u \text {, brain }}=0.2 \mathrm{~mL} \mathrm{gbr}^{-1}$, corresponding to the volume of interstitial fluid per gram in the rat brain. If the solute is uniformly distributed between the interstitial fluid and the intracellular fluid then $V_{u \text {, brain }}=0.8 \mathrm{~mL} \mathrm{gbr}^{-1}$, corresponding to the volume of water per gram in the rat brain. Values of $V_{u \text {,brain }}>0.8 \mathrm{~mL} \mathrm{gbr}^{-1}$, correspond to the case where the solute has affinity for brain tissue.

\section{THE BLOOD-BRAIN BARRIER AND DISEASE}

The barrier function of the BBB is critical for regulating transport to the brain, but also represents a significant roadblock in delivering drugs to the brain. Central nervous system diseases include mental disorders, migraine, epilepsy, neurodegenerative disease (e.g., Alzheimer's, Parkinson's, ALS, Huntington's), cerebrovascular disease (e.g., stroke), cancer, inflammatory disease (e.g., MS), trauma, and infections (e.g., meningitis; Hawkins and Davis, 2005; Hirtz et al., 2007; Neuwelt et al., 2008, 2011; Abbott et al., 2010; Daneman, 2012). Only very few CNS disorders such as depression, schizophrenia, chronic pain, and epilepsy are currently treatable with small molecule drug therapy. The BBB is the major roadblock in developing therapies for neurodegenerative diseases, cerebrovascular disease, inflammatory disease, infections, trauma, and brain cancer (de Boer and Gaillard, 2007; Pardridge, 2010).

Since the BBB is critical to maintain homeostasis in the brain, disruption can lead to changes in permeability, modulation of immune cell transport, and trafficking of pathogens into the brain (Hawkins and Davis, 2005; Engelhardt, 2008a,b; Abbott et al., 2010; Neuwelt et al., 2011). Disruption of the BBB is associated with many diseases of the central nervous system, including neurodegenerative diseases [e.g., Alzheimer's disease (Kalaria, 1999; Zlokovic, 2005; Desai et al., 2007; Zipser et al., 2007; Meyer et al., 2008; Hartz et al., 2010), ALS (Zhong et al., 2008), and Parkinson's disease (Kortekaas et al., 2005; Desai et al., 2007; Bartels et al., 2008)], cerebrovascular diseases [e.g., stroke (Belayev et al., 1996; Lippoldt et al., 2000; Lo et al., 2003; Del Zoppo, 2010; Moskowitz et al., 2010)], epilepsy and seizures (Seiffert et al., 2004; Oby and Janigro, 2006; Remy and Beck, 2006), brain infections [e.g., HIV encephalitis (Dallasta et al., 1999; Berger and Avison, 2004; Persidsky et al., 2006; Ivey et al., 2009) and meningitis (Uchiyama et al., 2009)], inflammatory diseases [e.g., MS (Kermode et al., 1990; Minagar and Alexander, 2003; Gold et al., 2006; Waubant, 2006; McQuaid et al., 2009)],

\section{REFERENCES}

Abbott, N. J. (2002). Astrocyteendothelial interactions and blood-brain barrier permeability. J. Anat. 200, 629-638. doi: 10.1046/j.1469-7580.2002.00064.x

Abbott, N. J., Patabendige, A. A., Dolman, D. E., Yusof, S. R., and Begley, D. J. (2010). Structure and function of the blood-brain barrier.

brain tumors (Davies, 2002; Papadopoulos et al., 2004; Bronger et al., 2005), and neurotrauma (Stahel et al., 2000; Kim and Dustin, 2006; Shlosberg et al., 2010). There is also emerging evidence that mental or psychological stress may lead to local disruption of the BBB (Friedman et al., 1996). The association of $\mathrm{BBB}$ disruption with $\mathrm{CNS}$ diseases, suggests that $\mathrm{BBB}$ repair may prove to be an effective approach to maintain health and aid recovery from disease, infection, or injury (Abbott et al., 2010).

\section{FUTURE PERSPECTIVES}

Drug delivery to the brain remains a major obstacle for treatment of CNS disorders. Advances in our understanding of the structure and function of the blood-brain barrier and development of innovative approaches for circumventing this barrier will be required to overcome the restricted access to brain circuits (Neuwelt et al., 2011). Moreover, there is an increasing appreciation that bloodbrain barrier disruption contributes to the progression of central nervous system diseases. A key challenge is in understanding the dynamic response of barrier elements to focal disruptions and in developing strategies to accelerate repair.

While there is emerging insight into the formation of the blood-brain barrier during development (Daneman et al., 2010; Sohet and Daneman, 2013), very little is known about how aging affects barrier function. This is important, as the greatest risk factor for neurodegenerative disorders is aging. Insight into the morphology, turnover, dynamic behavior, and mechanical properties of endothelial cells during aging, as well as functional interactions with other cell types in the neurovascular unit will be required to define the role of BBB changes in both age-dependent cognitive decline and the progression of neurodegenerative diseases.

Central to advances in our scientific understanding of the BBB will be improved models for scientific and translational research. From an engineering perspective, the key features of the neurovascular unit are: (1) BMECs that function in a cylindrical geometry and experience shear stress resulting from blood flow, (2) functional interactions between BMECs, astrocytes, pericytes, other glial cells and neurons, (3) blood, which contains multiple cell types and soluble factors, and (4) 3D extracellular matrix and basement membrane. Determining how these features of the microvasculature interact will aid in the generation of BBB models compatible with high throughput screening methods that are likely to be crucial to the development of novel therapeutics.

\section{SUPPLEMENTARY MATERIAL}

The Supplementary Material for this article can be found online at: http://www.frontiersin.org/Neuroengineering/10.3389/ fneng.2013.00007/abstract

R., Audus, K. L., et al. (1994). Quantitative approaches to delineate paracellular diffusion in cultured epithelial cell monolayers. J. Pharm. Sci. 83, 1529-1536. doi: 10.1002/jps.26008 31103

Aiello, L. C., and Dunbar, R. I. M. (1993). Neocortex size, group-size, and the evolution of language.
Curr. Anthropol. 34, 184-193. doi: $10.1086 / 204160$

Aiello, L. C., and Wheeler, P. (1995). The expensive-tissue hypothesis the brain and the digestive-system in human and primate evolution. Curr. Anthropol. 36, 199-221. doi: $10.1086 / 204350$

Aird, W. C. (2005). Spatial and temporal dynamics of the 
endothelium. J. Thromb. Haemost. 3, 1392-1406. doi: 10.1111/j.1538-7836.2005.01328.x

Aird, W. C. (2007). Phenotypic heterogeneity of the endothelium: I. Structure, function, and mechanisms. Circ. Res. 100, 158-173. doi: 10.1161/01.RES.0000255691. 76142.4a

Ananthanarayanan, B., Kim, Y., and Kumar, S. (2011). Elucidating the mechanobiology of malignant brain tumors using a brain matrix-mimetic hyaluronic acid hydrogel platform. Biomaterials 32, 7913-7923. doi: 10.1016/j.biomaterials.2011.07.005

Armulik, A., Abramsson, A., and Betsholtz, C. (2005). Endothelial/pericyte interactions. Circ. Res. 97, 512-523. doi: 10. 1161/01.RES.0000182903.16652.d7

Armulik, A., Genove, G., Mae, M., Nisancioglu, M. H., Wallgard, E., Niaudet, C., et al. (2010). Pericytes regulate the blood-brain barrier. Nature 468, 557-561. doi: 10.1038/nature09522

Artursson, P. (1991). Cell-cultures as models for drug absorption across the intestinal-mucosa. Crit. Rev. Ther. Drug Carrier Syst. 8, 305-330.

Artursson, P., Palm, K., and Luthman, K. (2001). Caco-2 monolayers in experimental and theoretical predictions of drug transport. Adv. Drug Deliv. Rev. 46, 27-43. doi: 10.1016/S0169-409X(00)00128-9

Asahara, T., Kawamoto, A., and Masuda, H. (2011). Concise review: circulating endothelial progenitor cells for vascular medicine. Stem Cells 29, 1650-1655. doi: 10.1002/stem.745

Attwell, D., Buchan, A. M., Charpak, S., Lauritzen, M., Macvicar, B. A., and Newman, E. A. (2010). Glial and neuronal control of brain blood flow. Nature 468, 232-243. doi: 10.1038/nature09613

Attwell, D., and Laughlin, S. B. (2001). An energy budget for signaling in the grey matter of the brain. J. Cereb. Blood Flow Metab. 21, 1133-1145. doi: 10.1097/00004647-20011000000001

Audus, K. L., and Borchardt, R. T. (1986). Characteristics of the large neutral amino acid transport system of bovine brain microvessel endothelial cell monolayers. J. Neurochem. 47, 484-488. doi: 10.1111/j.14714159.1986.tb04527.x

Aumailley, M., Bruckner-Tuderman, L., Carter, W. G., Deutzmann, R., Edgar, D., Ekblom, P., et al. (2005) A simplified laminin nomenclature.
Matrix Biol. 24, 326-332. doi: 10.1016/j.matbio.2005.05.006

Avdeef, A. (2001). Physicochemical profiling (solubility, permeability and charge state). Curr. Top. Med. Chem. 1, 277-351. doi: $10.2174 / 1568026013395100$

Avdeef, A. (2003). Absorption and Drug Development: Solubility, Permeability, and Charge State. Hoboken, NJ: Wiley. doi: 10.1002/047145026X

Avdeef, A., Artursson, P., Neuhoff, S., Lazorova, L., Gråsjö, J., and Tavelin, S. (2005). Caco-2 permeability of weakly basic drugs predicted with the Double-Sink PAMPA method. Eur. J. Pharm. Sci. 24, 333-349. doi: 10.1016/j.ejps.2004.11.011

Balimane, P. V., Han, Y. H., and Chong, S. H. (2006). Current industrial practices of assessing permeability and P-glycoprotein interaction. AAPS J. 8, E1-E13. doi: 10.1208/aapsj080101

Barry, P. H., and Diamond, J. M. (1984). Effects of unstirred layers on membrane phenomena. Physiol. Rev. 64, 763-872.

Bartels, A. L., Willemsen, A. T., Kortekaas, R., De Jong, B. M., De Vries, R., De Klerk, O., et al. (2008). Decreased blood-brain barrier P-glycoprotein function in the progression of Parkinson's disease, PSP and MSA. J. Neural Transm. 115, 1001-1009. doi: 10.1007/s00702-008-0030-y

Bauer, B., Hartz, A. M., Lucking, J. R., Yang, X., Pollack, G. M., and Miller, D. S. (2008). Coordinated nuclear receptor regulation of the efflux transporter, Mrp2, and the phaseII metabolizing enzyme, GSTpi, at the blood-brain barrier. J. Cereb. Blood Flow Metab. 28, 1222-1234. doi: $10.1038 / j \mathrm{cbfm} .2008 .16$

Bauer, B., Hartz, A. M., and Miller, D. S. (2007). Tumor necrosis factor alpha and endothelin-1 increase Pglycoprotein expression and transport activity at the blood-brain barrier. Mol. Pharmacol. 71, 667-675. doi: 10.1124/mol.106.029512

Bauer, B., Hartz, A. M. S., Fricker, G., and Miller, D. S. (2005). Modulation of p-glycoprotein transport function at the blood-brain barrier. Exp. Biol. Med. 230, 118-127.

Bazzoni, G., and Dejana, E. (2004). Endothelial cell-to-cell junctions: molecular organization and role in vascular homeostasis. Physiol. Rev. 84, 869-901. doi: 10.1152/physrev.00035.2003

Begley, D. J., and Brightman, M. W. (2003). "Structural and functional aspects of the blood brain barrier," in Progress in Drug Research, eds L. Prokai and K. Prokai-Tatrai (Basel:Birkhauser Verlag), 39-78.

Belayev, L., Busto, R., Zhao, W. and Ginsberg, M. D. (1996). Quantitative evaluation of bloodbrain barrier permeability following middle cerebral artery occlusion in rats. Brain Res. 739, 88-96. doi: 10.1016/S0006-8993 (96)00815-3

Bell, R. D., Winkler, E. A., Sagare, A. P., Singh, I., Larue, B., Deane, R., et al. (2010). Pericytes control key neurovascular functions and neuronal phenotype in the adult brain and during brain aging. Neuron 68, 409-427. doi: 10.1016/j.neuron.2010.09.043

Benarroch, E. E. (2011). Circumventricular organs: receptive and homeostatic functions and clinical implications. Neurology 77, 1198-1204. doi: 10.1212/WNL.0b013e31822f04a0

Berger, J. R., and Avison, M. (2004). The blood brain barrier in HIV infection. Front. Biosci. 9, 2680-2685. doi: 10.2741/1427

Bernas, M. J., Cardoso, F. L., Daley, S. K., Weinand, M. E., Campos, A. R., Ferreira, A. J. G., et al. (2010). Establishment of primary cultures of human brain microvascular endothelial cells to provide an in vitro cellular model of the blood-brain barrier. Nat. Protoc. 5, 1265-1272. doi: 10.1038/nprot.2010.76

Boero, J. A., Ascher, J., Arregui, A., Rovainen, C., and Woolsey, T. A. (1999). Increased brain capillaries in chronic hypoxia. J. Appl. Physiol. $86,1211-1219$.

Bonkowski, D., Katyshev, V., Balabanov, R. D., Borisov, A., and Dore-Duffy, P. (2011). The CNS microvascular pericyte: pericyte-astrocyte crosstalk in the regulation of tissue survival. Fluids Barriers CNS 8, 8. doi: 10.1186/2045-8118-8-8

Borowsky, I. W., and Collins, R. C. (1989a). Metabolic anatomy of brain - a comparison of regional capillary density, glucosemetabolism, and enzyme-activities. J. Comp. Neurol. 288, 401-413. doi: 10.1002/cne. 902880304

Borowsky, I. W., and Collins, R. C. (1989b). Metabolic anatomy of brain: a comparison of regional capillary density, glucose metabolism, and enzyme activities. J. Comp. Neurol. 288, 401-413. doi: $10.1002 /$ cne. 902880304

Bostrom, E., Hammarlund-Udenaes, M., and Simonsson, U. S. (2008). Blood-brain barrier transport helps to explain discrepancies in in vivo potency between oxycodone and morphine. Anesthesiology 108, 495-505. doi: 10.1097/ALN.0b013e318164cf9e

Bouw, M. R., Gardmark, M., and Hammarlund-Udenaes, M. (2000). Pharmacokineticpharmacodynamic modelling of morphine transport across the blood-brain barrier as a cause of the antinociceptive effect delay in rats - a microdialysis study. Pharm. Res. 17, 1220-1227. doi: 10.1023/A:1026414713509

Bowman, P. D., Ennis, S. R., Rarey, K. E., Lorris Betz, A., and Goldstein, G. W. (1983). Brain microvessel endothelial cells in tissue culture: a model for study of blood-brain barrier permeability. Ann. Neurol. 14, 396-402. doi: 10.1002/ana.410140403

Bradbury, M. W. (1993). The bloodbrain barrier. Exp. Physiol. 78, 453-472.

Brightman, M. W. (1977). Morphology of blood-brain interfaces. Exp. Eye Res. 25, 1-25. doi: 10.1016/S00144835(77)80008-0

Bronger, H., Konig, J., Kopplow, K., Steiner, H. H., Ahmadi, R., Herold-Mende, C., et al. (2005). ABCC drug efflux pumps and organic anion uptake transporters in human gliomas and the blood-tumor barrier. Cancer Res. 65, 11419-11428. doi 10.1158/0008-5472.CAN-05-1271

Butt, A. M., and Jones, H. C. (1992). Effect of histamine and antagonists on electrical-resistance across the blood-brain-barrier in rat brain-surface microvessels. Brain Res. 569, 100-105. doi: 10.1016/0006-8993(92)90374-I

Campos, C. R., Schroter, C., Wang, X., and Miller, D. S. (2012). $\mathrm{ABC}$ transporter function and regulation at the blood-spinal cord barrier. J. Cereb. Blood Flow Metab. 32, 1559-1566. doi: 10.1038/jcbfm.2012.47

Cannon, R. E., Peart, J. C., Hawkins, B. T., Campos, C. R., and Miller, D. S. (2012). Targeting blood-brain barrier sphingolipid signaling reduces basal P-glycoprotein activity and improves drug delivery to the brain. Proc. Natl. Acad. Sci. U.S.A. 109, 15930-15935. doi: 10.1073/pnas.1203534109

Caplan, B. A., Gerrity, R. G., and Schwartz, C. J. (1974). Endothelial cell morphology in focal areas of in vivo Evans blue uptake in the young pig aorta. I. Quantitative light microscopic findings. Exp. Mol. Pathol. 21, 102-117. doi: 10.1016/0014-4800(74)90082-3 
Cecchelli, R., Berezowski, V., Lundquist, S., Culot, M., Renftel, M., Dehouck, M. P., et al. (2007). Modelling of the blood-brain barrier in drug discovery and development. Nat. Rev. Drug Discov. 6, 650-661. doi: 10.1038/ $\operatorname{nrd} 2368$

Cecchelli, R., Dehouck, B., Descamps, L., Fenart, L., Buée-Scherrer, V., Duhem, C., et al. (1999). In vitro model for evaluating drug transport across the blood-brain barrier. $A d v$. Drug Deliv. Rev. 36, 165-178. doi: 10.1016/S0169-409X(98)00083-0

Chappa, A., Audus, K., and Lunte, S. (2006). Characteristics of substance p transport across the blood-brain barrier. Pharm. Res. 23, 1201-1208. doi: 10.1007/s11095-006-0068-1

Chien, S. (2007). Mechanotransduction and endothelial cell homeostasis: the wisdom of the cell. Am. J. Physiol. Heart Circ. Physiol. 292, H1209-H1224. doi: 10.1152/ajpheart.01047.2006

Chishty, M., Reichel, A., Siva, J., Abbott, N. J., and Begley, D. J. (2001). Affinity for the P-glycoprotein efflux pump at the blood-brain barrier may explain the lack of CNS sideeffects of modern antihistamines. J. Drug Target. 9, 223-228. doi: $10.3109 / 10611860108997930$

Conway, D., and Schwartz, M. A. (2012). Lessons from the endothelial junctional mechanosensory complex. F1000 Biol. Rep. 4, 1.

Crone, C. (1963). The permeability of capillaries in various organs as determined by use of the indicator diffusion method. Acta Physiol. Scand. 58, 292-305. doi: 10.1111/j.17481716.1963.tb02652.x

Crone, C., and Olesen, S. P. (1982). Electrical resistance of brain microvascular endothelium. Brain Res. 241, 49-55. doi: 10.1016/0006-8993(82)91227-6

Cucullo, L., Hossain, M., Puvenna, V., Marchi, N., and Janigro, D. (2011). The role of shear stress in blood-brain barrier endothelial physiology. BMC Neurosci. 12:40. doi: 10.1186/1471-2202-12-40

Dalkara, T., Gursoy-Ozdemir, Y., and Yemisci, M. (2011). Brain microvascular pericytes in health and disease. Acta Neuropathol. 122, 1-9. doi: 10.1007/s00401-011-0847-6

Dallasta, L. M., Pisarov, L. A., Esplen, J. E., Werley, J. V., Moses, A. V., Nelson, J. A., et al. (1999). Blood-brain barrier tight junction disruption in human immunodeficiency virus-1 encephalitis. Am. J. Pathol. 155, 1915-1927. doi: 10.1016/S0002-9440(10)65511-3
Daneman, R. (2012). The blood-brain barrier in health and disease. Ann. Neurol. 72, 648-672. doi: 10.1002/ana.23648

Daneman, R., Agalliu, D., Zhou, L., Kuhnert, F., Kuo, C. J., and Barres, B. A. (2009). Wnt/beta-catenin signaling is required for CNS, but not non-CNS, angiogenesis. Proc. Natl. Acad. Sci. U.S.A. 106, 641-646. doi: 10.1073/pnas.0805165106

Daneman, R., Zhou, L., Kebede, A. A., and Barres, B. A. (2010). Pericytes are required for blood-brain barrier integrity during embryogenesis. Nature 468, 562-566. doi: 10.1038/nature09513

Davies, D. C. (2002). Blood-brain barrier breakdown in septic encephalopathy and brain tumours. J. Anat. 200, 639-646. doi: 10.1046/j.1469-7580.2002.00065.x

Dawson, D. (1991). "Principles of membrane transport," in Handbook of Physiology, Section 6: The Gastrointestinal System, ed B. Rauner (Bethesda, MD: American Physiological Society), 1-45.

de Boer, A. G., and Gaillard, P. J. (2007). Drug targeting to the brain. Annu. Rev. Pharmacol. Toxicol. 47, 323-355. doi: 10.1146/annurev. pharmtox.47.120505.105237

de Boer, A. G., Van Der Sandt, I. C. J., and Gaillard, P. J. (2003). The role of drug transporters at the blood-brain barrier. Annu. Rev. Pharmacol. Toxicol. 43, 629-656. doi: 10.1146/annurev.pharmtox.43. 100901.140204

debault, L. E., and Cancilla, P. A. (1980). Gamma-glutamyltransferase transpeptidase in isolated brain endothelialcells - induction by glial-cells invitro. Science 207, 653-655. doi: 10.1126/science.6101511

Dejana, E. (2004). Endothelial cellcell junctions: happy together. Nat. Rev. Mol. Cell Biol. 5, 261-270. doi: 10.1038/nrm1357

Del Zoppo, G. J. (2010). The neurovascular unit in the setting of stroke. J. Intern. Med. 267, 156-171. doi: 10.1111/j.1365-2796.2009.02199.x

Deli, M. A., Abraham, C. S., Kataoka, Y., and Niwa, M. (2005). Permeability studies on in vitro blood-brain barrier models: physiology, pathology, and pharmacology. Cell. Mol. Neurobiol. 25, 59-127. doi: 10.1007/s10571-004-1377-8

Demeule, M., Regina, A., Jodoin, J., Laplante, A., Dagenais, C., Berthelet, F., et al. (2002). Drug transport to the brain: key roles for the efflux pump P-glycoprotein in the blood-brain barrier. Vascul.
Pharmacol. 38, 339-348. doi: 10.1016/S1537-1891(02)00201-X

Desai, B. S., Monahan, A. J., Carvey, P. M., and Hendey, B. (2007). Blood-brain barrier pathology in Alzheimer's and Parkinson's disease: implications for drug therapy. Cell Transplant. 16, 285-299.

Didier, A., Tiberghien, F., Wenger, R., and Loor, F. (1996). Detection of P-glycoprotein expression by tumoral cells with NBDL-CsA, a fluorescent derivative of cyclosporin A. Anticancer Drugs 7, 257-265. doi: 10.1097/00001813-19960500000004

Dolan, J. M., Kolega, J., and Meng, H. (2013). High wall shear stress and spatial gradients in vascular pathology: a review. Ann. Biomed. Eng. 41, 1411-1427. doi 10.1007/s10439-012-0695-0

Duvernoy, H., Delon, S., and Vannson, J. L. (1983). The vascularization of the human cerebellar cortex. Brain Res. Bull. 11, 419-480. doi 10.1016/0361-9230(83)90116-8

Duvernoy, H. M., and Risold, P. Y. (2007). The circumventricular organs: an atlas of comparative anatomy and vascularization. Brain Res. Rev. 56, 119-147. doi 10.1016/j.brainresrev.2007.06.002

Ekstrand, J., Hellsten, J., and Tingstrom, A. (2008). Environmental enrichment, exercise and corticosterone affect endothelial cell proliferation in adult rat hippocampus and prefrontal cortex. Neurosci. Lett. 442, 203-207. doi 10.1016/j.neulet.2008.06.085

Enerson, B. E., and Drewes, L. R. (2006). The rat blood-brain barrier transcriptome. J. Cereb. Blood Flow Metab. 26, 959-973. doi: 10.1038/sj.jcbfm. 9600249

Engelhardt, B. (2008a). The bloodcentral nervous system barriers actively control immune cell entry into the central nervous system. Curr. Pharm. Des. 14, 1555-1565. doi: $10.2174 / 138161208784705432$

Engelhardt, B. (2008b). Immune cell entry into the central nervous system: involvement of adhesion molecules and chemokines. J. Neurol. Sci. 274, 23-26. doi: 10.1016/j.jns.2008.05.019

Fahraeus, R., and Lindqvist, T. (1931). The viscosity of the blood in narrow capillary tubes. Am. J. Physiol. 96, 562-568.

Farkas, E., and Luiten, P. G. M. (2001). Cerebral microvascular pathology in aging and Alzheimer's disease. Prog. Neurobiol. 64, 575-611. doi 10.1016/S0301-0082(00)00068-X

Fenstermacher, J., Gross, P., Sposito, N., Acuff, V., Pettersen, S., and Gruber,
K. (1988). Structural and functional variations in capillary systems within the brain. Ann. N.Y. Acad. Sci. 529, 21-30. doi: 10.1111/j.17496632.1988.tb51416.x

Fernandez-Klett, F., Offenhauser, N., Dirnagl, U., Priller, J., and Lindauer, U. (2010). Pericytes in capillaries are contractile in vivo, but arterioles mediate functional hyperemia in the mouse brain. Proc. Natl. Acad. Sci. U.S.A. 107, 22290-22295. doi: 10.1073/pnas.1011321108

Fiacco, T. A., Agulhon, C., and McCarthy, K. D. (2009). Sorting out astrocyte physiology from pharmacology. Annu. Rev. Pharmacol. Toxicol. 49, 151-174. doi: 10.1146/ annurev.pharmtox.011008.145602

Fish, J. L., and Lockwood, C. A. (2003). Dietary constraints on encephalization in primates. Am. Phys. Anthropol. 120, 171-181. doi: 10.1002/ajpa.10136

Fisher, M. (2009). Pericyte signaling in the neurovascular unit. Stroke 40, S13-15. doi: 10.1161/STROKEAHA.108.533117

Franke, H., Galla, H.-J., and Beuckmann, C. T. (1999). An improved low-permeability in vitro-model of the bloodbrain barrier: transport studies on retinoids, sucrose, haloperidol, caffeine and mannitol. Brain Res. 818, 65-71. doi: 10.1016/S0006-8993(98)01282-7

Freed, A. L., Audus, K. L., and Lunte, S. M. (2001). Investigation of the metabolism of substance $\mathrm{P}$ at the blood-brain barrier using capillary electrophoresis with laserinduced fluorescence detection. Electrophoresis 22, 3778-3784.

Freeman, M. R. (2010). Specification and morphogenesis of astrocytes. Science 330, 774-778. doi: 10.1126/science.1190928

Friedman, A., Kaufer, D., Shemer, J., Hendler, I., Soreq, H., and TurKaspa, I. (1996). Pyridostigmine brain penetration under stress enhances neuronal excitability and induces early immediate transcriptional response. Nat. Med. 2, 1382-1385. doi: 10.1038/nm1296-1382

Fujikawa, M., Nakao, K., Shimizu, R., and Akamatsu, M. (2007). QSAR study on permeability of hydrophobic compounds with artificial membranes. Bioorg. Med. Chem. 15, 3756-3767. doi: 10.1016/j.bmc.2007.03.040

Furuse, M., and Tsukita, S. (2006). Claudins in occluding junctions of humans and flies. Trends Cell Biol. 16, 181-188. doi: 10.1016/j.tcb.2006.02.006 
Ganong, W. F. (2000). Circumventricular organs: definition and role in the regulation of endocrine and autonomic function. Clin. Exp. Pharmacol. Physiol. 27, 422-427. doi: 10.1046/j.1440-1681.2000.03259.x

Giacomini, K. M., Huang, S. M., Tweedie, D. J., Benet, L. Z., Brouwer, K. L., Chu, X., et al. (2010). Membrane transporters in drug development. Nat. Rev. Drug Discov. 9, 215-236. doi: 10.1038/nrd3028

Gold, R., Linington, C., and Lassmann, H. (2006). Understanding pathogenesis and therapy of multiple sclerosis via animal models: 70 years of merits and culprits in experimental autoimmune encephalomyelitis research. Brain 129, 1953-1971. doi: 10.1093/brain/awl075

Gratton, J. A., Abraham, M. H., Bradbury, M. W., and Chadha, H. S. (1997). Molecular factors influencing drug transfer across the blood-brain barrier. J. Pharm. Pharmacol. 49, 1211-1216. doi: 10.1111/j.20427158.1997.tb06072.x

Gross, P. M., Sposito, N. M., Pettersen, S. E., and Fenstermacher, J. D. (1986). Differences in function and structure of the capillary endothelium in gray matter, white matter and a circumventricular organ of rat brain. Blood Vessels 23, 261-270.

Gumbleton, M., and Audus, K. L. (2001). Progress and limitations in the use of in vitro cell cultures to serve as a permeability screen for the blood-brain barrier. J. Pharm. Sci. 90, 1681-1698. doi: 10.1002/jps.1119

Hahn, C., and Schwartz, M. A. (2009). Mechanotransduction in vascular physiology and atherogenesis. Nat. Rev. Mol. Cell Biol. 10, 53-62. doi: 10.1038/nrm2596

Halassa, M. M., and Haydon, P. G. (2010). Integrated brain circuits: astrocytic networks modulate neuronal activity and behavior. Annu. Rev. Physiol. 72, 335-355. doi 10.1146/annurev-physiol-021909135843

Hamilton, N. B., Attwell, D., and Hall, C. N. (2010). Pericyte-mediated regulation of capillary diameter: a component of neurovascular coupling in health and disease. Front. Neuroenergetics 2:5. doi: 10.3389/fnene.2010.00005

Hammarlund-Udenaes, M., Bredberg, U., and Friden, M. (2009). Methodologies to assess brain drug delivery in lead optimization. Curr. Top. Med. Chem. 9, 148-162. doi: $10.2174 / 156802609787521607$
Hammarlund-Udenaes, M., Friden, M., Syvanen, S., and Gupta, A. (2008). On the rate and extent of drug delivery to the brain. Pharm. Res. 25, 1737-1750. doi: 10.1007/s11095-007-9502-2

Hartmann, C., Zozulya, A., Wegener, J., and Galla, H. J. (2007). The impact of glia-derived extracellular matrices on the barrier function of cerebral endothelial cells: an in vitro study. Exp. Cell Res. 313, 1318-1325. doi: 10.1016/j.yexcr. 2007.01.024

Hartz, A. M., and Bauer, B. (2011). $\mathrm{ABC}$ transporters in the CNS - an inventory. Curr. Pharm. Biotechnol. 12， 656-673. doi 10.2174/138920111795164020

Hartz, A. M., Miller, D. S., and Bauer, B. (2010). Restoring blood-brain barrier P-glycoprotein reduces brain amyloid-beta in a mouse model of Alzheimer's disease. Mol. Pharmacol. 77, 715-723. doi: 10.1124/mol.109.061754

Hartz, A. M. S., Bauer, B., Fricker, G., and Miller, D. S. (2004). Rapid regulation of p-glycoprotein at the blood-brain barrier by endothelin1. Mol. Pharmacol. 66, 387-394. doi: 10.1124/mol.104.001503

Haseloff, R. F., Blasig, I. E., Bauer, H. C., and Bauer, H. (2005). In search of the astrocytic factor(s) modulating blood-brain barrier functions in brain capillary endothelial cells in vitro. Cell. Mol. Neurobiol. 25, 25-39. doi: 10.1007/s10571-004$1375-\mathrm{x}$

Hatherell, K., Couraud, P. O., Romero, I. A., Weksler, B., and Pilkington, G. J. (2011). Development of a three-dimensional, allhuman in vitro model of the blood-brain barrier using mono-, co-, and tri-cultivation Transwell models. J. Neurosci. Methods 199, 223-229. doi: 10.1016/j.jneumeth.2011.05.012

Hauck, E. F., Apostel, S., Hoffmann, J. F., Heimann, A., and Kempski, O. (2004). Capillary flow and diameter changes during reperfusion after global cerebral ischemia studied by intravital video microscopy. J. Cereb. Blood Flow Metab. 24, 383-391. doi: 10.1097/00004647-20040400000003

Hawkins, B. T., and Davis, T. P. (2005). The blood-brain barrier/neurovascular unit in health and disease. Pharmacol. Rev. 57, 173-185. doi: 10.1124/pr.57.2.4

Hawkins, B. T., Sykes, D. B., and Miller, D. S. (2010). Rapid, reversible modulation of blood-brain barrier pglycoprotein transport activity by vascular endothelial growth factor.
J. Neurosci. 30, 1417-1425. doi 10.1523/JNEUROSCI.5103-09.2010

Hawkins, R. A., Peterson, D. R., and Vina, J. R. (2002). The complementary membranes forming the blood-brain barrier. IUBMB Life 54, 101-107. doi: $10.1080 / 15216540214541$

Heinzer, S., Kuhn, G., Krucker, T., Meyer, E., Ulmann-Schuler, A., Stampanoni, M., et al. (2008). Novel three-dimensional analysis tool for vascular trees indicates complete micro-networks, not single capillaries, as the angiogenic endpoint in mice overexpressing human VEGF(165) in the brain Neuroimage 39, 1549-1558. doi 10.1016/j.neuroimage.2007.10.054

Hewitt, K. J., Agarwal, R., and Morin, P. J. (2006). The claudin gene family: expression in normal and neoplastic tissues. BMC Cancer 6:186. doi 10.1186/1471-2407-6-186

Hirtz, D., Thurman, D. J., GwinnHardy, K., Mohamed, M. Chaudhuri, A. R., and Zalutsky, R. (2007). How common are the "common" neurologic disorders? Neurology 68, 326-337. doi: 10 . 1212/01.wnl.0000252807.38124.a3

Hobson, B., and Denekamp, J. (1984). Endothelial proliferation in tumours and normal tissues: continuous labelling studies. Br. J. Cancer 49, 405-413. doi 10.1038/bjc. 1984.66

Holliday, M. A. (1986). "Body composition and energy needs during growth," in Human Growth: A Comprehensive Treatise, eds $\mathrm{F}$ Falkner and J. M. Tanner (Newyork, NY: Plenum), 101-117.

Hossmann, K. A. (2006) Pathophysiology and therapy of experimental stroke. Cell. Mol. Neurobiol. 26, 1057-1083. doi 10.1007/s10571-006-9008-1

Hubatsch, I., Ragnarsson, E. G., and Artursson, P. (2007) Determination of drug permeability and prediction of drug absorption in Caco-2 monolayers. Nat. Protoc. 2, 2111-2119. doi 10.1038/nprot.2007.303

Iadecola, C. (2004). Neurovascular regulation in the normal brain and in Alzheimer's disease. Nat Rev. Neurosci. 5, 347-360. doi 10.1038/nrn1387

Iadecola, C., and Nedergaard, M. (2007). Glial regulation of the cerebral microvasculature. Nat. Neurosci. 10, 1369-1376. doi $10.1038 / \mathrm{nn} 2003$

Ito, H., Kanno, I., Ibaraki, M. Hatazawa, J., and Miura, S. (2003). Changes in human cerebral blood flow and cerebral blood volume during hypercapnia and hypocapnia measured by positron emission tomography. J. Cereb. Blood Flow Metab. 23, 665-670. doi: 10.1097/ 01.WCB.0000067721.64998.F5

Ivey, N. S., Maclean, A. G., and Lackner, A. A. (2009). Acquired immunodeficiency syndrome and the blood-brain barrier. J. Neurovirol. 15, 111-122. doi: $10.1080 / 13550280902769764$

Janzer, R. C., and Raff, M. C. (1987). Astrocytes induce blood brain barrier properties in endothelial cells. Nature 325, 253-257. doi: 10.1038/325253a0

Johnson, B. D., Mather, K. J., and Wallace, J. P. (2011) Mechanotransduction of shear in the endothelium: basic studies and clinical implications Vasc. Med. 16, 365-377. doi: 10.1177/1358863X11422109

Kalaria, R. N. (1999). The bloodbrain barrier and cerebrovascular pathology in Alzheimer's disease. Ann. N.Y. Acad. Sci. 893, 113-125. doi: 10.1111/j.17496632.1999.tb07821.x

Kamiya, A., Bukhari, R., and Togawa, T. (1984). Adaptive regulation of wall shear stress optimizing vascular tree function. Bull. Math. Biol. 46, 127-137.

Karbowski, J. (2007). Global and regional brain metabolic scaling and its functional consequences. BMC Biol. 5:18. doi: 10.1186/1741-7007-5-18

Karbowski, J. (2009). Thermodynamic constraints on neural dimensions, firing rates, brain temperature and size. J. Comput. Neurosci. 27, 415-436. doi: 10.1007/s10827-009-0153-7

Karbowski, J. (2011). Scaling of brain metabolism and blood flow in relation to capillary and neural scaling. PLoS ONE 6:e26709. doi: 10.1371/journal.pone.0026709

Karlsson, J., and Artursson, P. (1991) A method for the determination of cellular permeability coefficients and aqueous boundary-layer thickness in monolayers of intestinal epithelial (caco-2) cells grown in permeable filter chambers. Int. I. Pharm. 71, 55-64. doi: 10.1016/0378-5173(91)90067-X

Kedem, O., and Katchalsky, A. (1958). Thermodynamic analysis of the permeability of biological membranes to non-electrolytes. Biochim. Biophys. Acta 27, 229-246. doi 10.1016/0006-3002(58)90330-5

Kermode, A. G., Thompson, A. J., Tofts, P., Macmanus, D. G., Kendall, B. E., Kingsley, D. P. E., et al. (1990). Breakdown of 
the blood-brain-barrier precedes symptoms and other mri signs of new lesions in multiple-sclerosis pathogenetic and clinical implications. Brain 113, 1477-1489. doi: 10.1093/brain/113.5.1477

Kim, J. H., Kim, J. H., Yu, Y. S., Kim, D. H., and Kim, K. W. (2009). Recruitment of pericytes and astrocytes is closely related to the formation of tight junction in developing retinal vessels. J. Neurosci. Res. 87, 653-659. doi: 10.1002/jnr.21884

Kim, J. V., and Dustin, M. L. (2006). Innate response to focal necrotic injury inside the blood-brain barrier. J. Immunol. 177, 5269-5277.

King, M., Su, W., Chang, A., Zuckerman, A., and Pasternak, G. W. (2001). Transport of opioids from the brain to the periphery by P-glycoprotein: peripheral actions of central drugs. Nat. Neurosci. 4, 268-274. doi: 10.1038/85115

Kinney, J. P., Spacek, J., Bartol, T. M., Bajaj, C. L., Harris, K. M., and Sejnowski, T. J. (2013). Extracellular sheets and tunnels modulate glutamate diffusion in hippocampal neuropil. J. Comp. Neurol. 521, 448-464. doi: $10.1002 / \mathrm{cne} .23181$

Kleiber, M. (1947). Body size and metabolic rate. Physiol. Rev. 27, 511-541.

Klein, B., Kuschinsky, W., Schrock, H., and Vetterlein, F. (1986). Interdependency of local capillary density, blood-flow, and metabolism in rat brains. Am. J. Physiol. 251, H1333-H1340.

Kortekaas, R., Leenders, K. L., Van Oostrom, J. C., Vaalburg, W., Bart, J., Willemsen, A. T., et al. (2005). Blood-brain barrier dysfunction in parkinsonian midbrain in vivo. Ann. Neurol. 57, 176-179. doi: 10.1002/ana.20369

Koutsiaris, A. G., Tachmitzi, S. V., Batis, N., Kotoula, M. G., Karabatsas, C. H., Tsironi, E., et al. (2007). Volume flow and wall shear stress quantification in the human conjunctival capillaries and post-capillary venules in vivo. Biorheology 44, 375-386.

Krause, G., Winkler, L., Piehl, C., Blasig, I., Piontek, J., and Muller, S. L. (2009). Structure and function of extracellular claudin domains. Ann. N.Y. Acad. Sci. 1165, 34-43. doi: 10.1111/j.1749-6632.2009.04057.x

Kreczmanski, P., Heinsen, H., Mantua, V., Woltersdorf, F., Masson, T., Ulfig, N., et al. (2009). Microvessel length density, total length, and length per neuron in five subcortical regions in schizophrenia. Acta Neuropathol. 117, 409-421. doi: 10.1007/s00401009-0482-7
Kreczmanski, P., Schmidt-Kastner, R., Heinsen, H., Steinbusch, H. W. M. Hof, P. R., and Schmitz, C. (2005). Stereological studies of capillary length density in the frontal cortex of schizophrenics. Acta Neuropathol. 109, 510-518. doi: 10.1007/s00401005-1003-y

Krencik, R., Weick, J. P., Liu, Y., Zhang, Z. J., and Zhang, S. C. (2011). Specification of transplantable astroglial subtypes from human pluripotent stem cells. Nat. Biotechnol. 29, 528-534. doi: 10.1038/nbt.1877

Krizanac-Bengez, L., Mayberg, M. R., and Janigro, D. (2004). The cerebral vasculature as a therapeutic target for neurological disorders and the role of shear stress in vascular homeostatis and pathophysiology. Neurol. Res. 26, 846-853. doi: 10.1179/016164104X3789

Kroeger, D., Tamburri, A., Amzica, F., and Sik, A. (2010). Activitydependent layer-specific changes in the extracellular chloride concentration and chloride driving force in the rat hippocampus. J. Neurophysiol. 103, 1905-1914. doi: 10.1152/jn.00497.2009

Krueger, M., and Bechmann, I. (2010). CNS pericytes: concepts, misconceptions, and a way out. Glia 58, 1-10. doi: 10.1002/glia.20898

Kubinyi, H. (1977). Quantitative structure-activity relationships. 7 . The bilinear model, a new model for nonlinear dependence of biological activity on hydrophobic character. J. Med. Chem. 20, 625-629. doi 10.1021/jm00215a002

Kuhnline Sloan, C. D., Nandi, P., Linz, T. H., Aldrich, J. V., Audus, K. L., and Lunte, S. M. (2012). Analytical and biological methods for probing the blood-brain barrier. Annu. Rev. Anal. Chem. (Palo Alto Calif) 5, 505-531. doi: 10.1146/annurevanchem-062011-143002

Laurent, T. C., and Fraser, J. R. E. (1992). Hyaluronan. FASEB J. 6, 2397-2404.

Lee, G., Dallas, S., Hong, M., and Bendayan, R. (2001). Drug transporters in the central nervous system: brain barriers and brain parenchyma considerations. Pharmacol. Rev. 53, 569-596.

Lee, S. W., Kim, W. J., Choi, Y. K., Song, H. S., Son, M. J., Gelman, I. H., et al. (2003). SSeCKS regulates angiogenesis and tight junction formation in blood-brain barrier. Nat. Med. 9 , 900-906. doi: 10.1038/nm889

Lennie, P. (2003). The cost of cortical computation. Curr. Biol. 13, 493-497. doi: 10.1016/S0960-9822(03)00135-0
Levesque, M. J., and Nerem, R. M. (1985). The elongation and orientation of cultured endothelialcells in response to shear-stress. J. Biomech. Eng. 107, 341-347. doi $10.1115 / 1.3138567$

Liddelow, S. A. (2011). Fluids and barriers of the CNS: a historical viewpoint. Fluids Barriers CNS 8, 2. doi: 10.1186/2045-8118-8-2

Lipinski, C. A., Lombardo, F., Dominy, B. W., and Feeney, P. J. (2001) Experimental and computational approaches to estimate solubility and permeability in drug discovery and development settings. $A d v$. Drug Deliv. Rev. 46, 3-26. doi 10.1016/S0169-409X(00)00129-0

Lippmann, E. S., Azarin, S. M., Kay, J. E., Nessler, R. A., Wilson, H. K., AlAhmad, A., et al. (2012). Derivation of blood-brain barrier endothelial cells from human pluripotent stem cells. Nat. Biotechnol. 30, 783-791. doi: $10.1038 /$ nbt.2247

Lippoldt, A., Kniesel, U., Liebner, S. Kalbacher, H., Kirsch, T., Wolburg, H., et al. (2000). Structural alterations of tight junctions are associated with loss of polarity in strokeprone spontaneously hypertensive rat blood-brain barrier endothelia cells. Brain Res. 885, 251-261. doi 10.1016/S0006-8993(00)02954-1

Liu, X., Tu, M., Kelly, R. S., Chen, C., and Smith, B. J. (2004). Development of a computational approach to predict blood-brain barrier permeability Drug Metab. Dispos. 32, 132-139. doi: $10.1124 / \mathrm{dmd} .32 .1 .132$

Lo, E. H., Dalkara, T., and Moskowitz, M. A. (2003). Mechanisms, challenges and opportunities in stroke. Nat. Rev. Neurosci. 4, 399-415. doi 10.1038/nrn1106

Madara, J. L. (1998). Regulation of the movement of solutes across tight junctions. Annu. Rev. Physiol. 60, 143-159. doi: 10.1146/annurev.physiol.60.1.143

Malek, A. M., and Izumo, S. (1996) Mechanism of endothelial cell shape change and cytoskeletal remodeling in response to fluid shear stress. J. Cell Sci. 109, 713-726.

McNaught, A. D., and Wilkinson, A. (1997). Compendium of Chemical Terminology: IUPAC Recommendations. Oxford: Blackwell Science.

McQuaid, S., Cunnea, P., McMahon, J., and Fitzgerald, U. (2009). The effects of blood-brain barrier disruption on glial cell function in multiple sclerosis. Biochem. Soc. Trans. 37, 329-331. doi 10.1042/BST0370329
Meyer, E. P., Ulmann-Schuler, A., Staufenbiel, M., and Krucker, T. (2008). Altered morphology and 3D architecture of brain vasculature in a mouse model for Alzheimer's disease. Proc. Natl. Acad. Sci. U.S.A. 105, 3587-3592. doi: 10.1073/pnas. 0709788105

Miller, D. S. (2003). Confocal imaging of xenobiotic transport across the blood-brain barrier. J. Exp. Zool. A Comp. Exp. Biol. 300A, 84-90. doi: 10.1002/jez.a.10313

Minagar, A., and Alexander, J. S. (2003). Blood-brain barrier disruption in multiple sclerosis. Mult. Scler. 9, 540-549. doi: $10.1191 / 1352458503 \mathrm{~ms} 965 \mathrm{oa}$

Morita, K., Sasaki, H., Furuse, M., and Tsukita, S. (1999). Endothelial claudin: claudin-5/TMVCF constitutes tight junction strands in endothelial cells. J. Cell Biol. 147, 185-194. doi: 10.1083/jcb.147.1.185

Moskowitz, M. A., Lo, E. H., and Iadecola, C. (2010). The science of stroke: mechanisms in search of treatments. Neuron 67, 181-198. doi: 10.1016/j.neuron.2010.07.002

Nag, S. (2003). "Morphological and molecular properties of cellular components of normal cerebra vessels," in The Blood-Brain Barrier: Biological and Research Protocols, ed S. Nag (New Jersey, NJ: Humana Press), 3-36.

Nakagawa, S., Deli, M. A., Kawaguchi, H., Shimizudani, T., Shimono, T., Kittel, A., et al. (2009). A new blood-brain barrier model using primary rat brain endothelia cells, pericytes and astrocytes. Neurochem. Int. 54, 253-263. doi: 10.1016/j.neuint.2008.12.002

Navarrete, A., van Schaik, C. P., and Isler, K. (2011). Energetics and the evolution of human brain size. Nature 480, 91-93. doi: 10.1038/nature10629

Nerem, R. M., Levesque, M. J., and Cornhill, J. F. (1981). Vascular endothelial morphology as an indicator of the pattern of blood-flow. J. Biomech. Eng. 103, 172-176. doi: 10.1115/1.3138275

Neuwelt, E., Abbott, N., Abrey, L. Banks, W. A., Blakley, B., Davis, T., et al. (2008). Strategies to advance translational research into brain barriers. Lancet Neurol. 7 84-96. doi: 10.1016/S14744422(07)70326-5

Neuwelt, E. A., Bauer, B., Fahlke, C. Fricker, G., Iadecola, C., Janigro, D., et al. (2011). Engaging neuroscience to advance translational research in brain barrier biology. Nat. Rev. Neurosci. 12, 169-182. doi: 10.1038/nrn2995 
Nicaise, C., Mitrecic, D., Demetter, P., De Decker, R., Authelet, M., Boom, A., et al. (2009). Impaired bloodbrain and blood-spinal cord barriers in mutant SOD1-linked ALS rat. Brain Res. 1301, 152-162. doi: 10.1016/j.brainres.2009.09.018

Nicholson, C. (2001). Diffusion and related transport mechanisms in brain tissue. Rep. Prog. Phys. 64, 815-884. doi: 10.1088/0034-4885/64/7/202

Nitta, T., Hata, M., Gotoh, S., Seo, Y., Sasaki, H., Hashimoto, N., et al. (2003). Size-selective loosening of the blood-brain barrier in claudin-5-deficient mice. J. Cell Biol. 161, 653-660. doi: 10.1083/jcb.200302070

Oberheim, N. A., Takano, T., Han, X., He, W., Lin, J. H. C., Wang, F., et al. (2009). Uniquely hominid features of adult human astrocytes. J. Neurosci. 29, 3276-3287. doi: 10.1523/JNEUROSCI.4707-08.2009

Oby, E., and Janigro, D. (2006). The blood-brain barrier and epilepsy. Epilepsia 47, 1761-1774. doi: 10.1111/j.1528-1167.2006.00817.x

Ohashi, T., and Sato, M. (2005). Remodeling of vascular endothelial cells exposed to fluid shear stress: experimental and numerical approach. Fluid Dyn. Res. 37, 40-59. doi: 10.1016/j.fluiddyn.2004.08.005

Ohno, K., Pettigrew, K. D., and Rapoport, S. I. (1978). Lower limits of cerebrovascular permeability to nonelectrolytes in the conscious rat. Am. J. Physiol. 235, H299-H307.

Ohtsuki, S., and Terasaki, T. (2007). Contribution of carrier-mediated transport systems to the bloodbrain barrier as a supporting and protecting interface for the brain; importance for CNS drug discovery and development. Pharm. Res. 24, 1745-1758. doi: 10.1007/s11095-007-9374-5

Papadopoulos, M. C., Saadoun, S., Binder, D. K., Manley, G. T., Krishna, S., and Verkman, A. S. (2004). Molecular mechanisms of brain tumor edema. Neuroscience 129, 1011-1020. doi: 10.1016/j.neuroscience.2004.05.044

Papaioannou, T. G., and Stefanadis, C. (2005). Vascular wall shear stress: basic principles and methods. Hellenic J. Cardiol. 46, 9-15.

Pardridge, W. M. (1998). CNS drug design based on principles of blood-brain barrier transport. J. Neurochem. 70, 1781-1792. doi: 10.1046/j.14714159.1998.70051781.x

Pardridge, W. M. (2003). Bloodbrain barrier drug targeting: the future of brain drug development. Mol. Interv. 3, 90-105. doi 10.1124/mi.3.2.90

Pardridge, W. M. (2005). The bloodbrain barrier: bottleneck in brain drug development. NeuroRx 2, 3-14. doi: 10.1602/neurorx.2.1.3

Pardridge, W. M. (2006). Molecular Trojan horses for blood-brain barrier drug delivery. Curr. Opin. Pharmacol. 6, 494-500. doi 10.1016/j.coph.2006.06.001

Pardridge, W. M. (2008). Reengineering biopharmaceuticals for delivery to brain with molecular Trojan horses. Bioconjug. Chem. 19, 1327-1338. doi: 10.1021/bc800148t

Pardridge, W. M. (2010). Biopharmaceutical drug targeting to the brain. J. Drug Target. 18, 157-167. doi: $10.3109 / 10611860903548354$

Pardridge, W. M., Triguero, D., Yang, J., and Cancilla, P. A. (1990). Comparison of in vitro and in vivo models of drug transcytosis through the blood-brain barrier. J. Pharmacol. Exp. Ther. 253, 884-891.

Pathak, A. P., Kim, E., Zhang, J., and Jones, M. V. (2011). Three-dimensional imaging of the mouse neurovasculature with magnetic resonance microscopy. PLoS ONE 6:e22643. doi: 10.1371/journal.pone.0022643

Peppiatt, C. M., Howarth, C., Mobbs, P., and Attwell, D. (2006). Bidirectional control of CNS capillary diameter by pericytes. Nature 443, 700-704. doi: 10.1038/nature05193

Persidsky, Y., Heilman, D., Haorah, J., Zelivyanskaya, M., Persidsky, R., Weber, G. A., et al. (2006). Rho-mediated regulation of tight junctions during monocyte migration across the blood-brain barrier in HIV-1 encephalitis (HIVE). Blood 107, 4770-4780. doi: 10.1182/blood-2005-11-4721

Pike, V. W. (2009). PET radiotracers: crossing the blood-brain barrier and surviving metabolism. Trends Pharmacol. Sci. 30, 431-440. doi: 10.1016/j.tips.2009.05.005

Piontek, J., Winkler, L., Wolburg, H., Muller, S. L., Zuleger, N., Piehl, C., et al. (2008). Formation of tight junction: determinants of homophilic interaction between classic claudins. FASEB J. 22, 146-158. doi: 10.1096/fj.07-8319com

Potts, R. (2011). Evolution big brains explained. Nature 480, 43-44. doi: $10.1038 / 480043 a$

Raichle, M. E., and Mintun, M. A. (2006). Brain work and brain imaging. Annu. Rev. Neurosci. 29, 449-476. doi: 10.1146/annurev. neuro.29.051605.112819

Rapoport, S. I., Ohno, K., and Pettigrew, K. D. (1979). Drug entry into the brain. Brain Res. 172, 354-359. doi 10.1016/0006-8993(79)90546-8

Reese, T. S., and Karnovsky, M. J. (1967). Fine structural localization of a blood-brain barrier to exogenous peroxidase. J. Cell Biol. 34, 207-217. doi: 10.1083/jcb. 34.1.207

Reichel, A., Begley, D. J., and Abbottt, N. J. (2003). "An overview of in vitro techniques for blood brain barrier studies," in Methods in Molecular Medicine, Vol. 89: The Blood Brain Barrier, ed S. Nag (Totowa, NJ: Humana), 307-324. doi: 10.1385/159259-419-0:307

Remy, S., and Beck, H. (2006). Molecular and cellular mechanisms of pharmacoresistance in epilepsy. Brain 129, 18-35. doi: 10.1093/brain/awh682

Renkin, E. M. (1959). Transport of potassium-42 from blood to tissue in isolated mammalian skeletal muscles. Am. J. Physiol. 197, 1205-1210.

Ribatti, D., Nico, B., Crivellato, E., and Artico, M. (2006). Development of the blood-brain barrier: a historical point of view. Anat. Rec. B New Anat. 289, 3-8. doi: 10.1002/ar.b.20087

Robey, R. W., Honjo, Y., Van De Laar, A., Miyake, K., Regis, J. T., Litman, T., et al. (2001). A functional assay for detection of the mitoxantrone resistance protein, MXR (ABCG2). Biochim. Biophys. Acta 1512, 171-182. doi: 10.1016/S00052736(01)00308-X

Roth, G., and Dicke, U. (2005) Evolution of the brain and intelligence. Trends Cogn. Sci. (Regul. Ed.) 9, 250-257. doi 10.1016/j.tics.2005.03.005

Sanes, J. R. (1989). Extracellularmatrix molecules that influence neural development. Annu. Rev. Neurosci. 12, 491-516. doi: 10.1146 annurev.ne.12.030189.002423

Sawada, T., Kato, Y., Sakayori, N., Takekawa, Y., and Kobayashi, M. (1999). Expression of the multidrug-resistance P-glycoprotein (Pgp, MDR-1) by endothelial cells of the neovasculature in central nervous system tumors. Brain Tumor Pathol. 16, 23-27. doi: 10.1007/BF02478898

Schlageter, K. E., Molnar, P., Lapin, G. D., and Groothuis, D. R. (1999). Microvessel organization and structure in experimental brain tumors: microvessel populations with distinctive structural and functional properties. Microvasc. Res. 58, 312-328. doi: 10.1006/mvre.1999. 2188

Schramm, U., Fricker, G., Wenger, R., and Miller, D. S. (1995) P-glycoprotein-mediated secretion of a fluorescent cyclosporin analogue by teleost renal proximal tubules. Am. J. Physiol. 268, F46-F52.

Seiffert, E., Dreier, J. P., Ivens, S., Bechmann, I., Tomkins, O., Heinemann, U., et al. (2004). Lasting blood-brain barrier disruption induces epileptic focus in the rat somatosensory cortex. J. Neurosci. 24, 7829-7836. doi: 10.1523/JNEUROSCI.1751-04.2004

Shah, M. V., Audus, K. L., and Borchardt, R. T. (1989). The Application of bovine brain microvessel endothelial-cell monolayers grown onto polycarbonate membranes in vitro to estimate the potential permeability of solutes through the blood-brain barrier. Pharm. Res. 6, 624-627. doi: 10.1023/A:1015913817221

Shen, L., Weber, C. R., Raleigh, D. R., Yu, D., and Turner, J. R. (2011). Tight junction pore and leak pathways: a dynamic duo. Annu. Rev. Physiol. 73, 283-309. doi: 10.1146/annurev-physiol-012110142150

Shepro, D., and Morel, N. M. (1993). Pericyte physiology. FASEB J. 7, 1031-1038.

Shlosberg, D., Benifla, M., Kaufer, D., and Friedman, A. (2010). Blood-brain barrier breakdown as a therapeutic target in traumatic brain injury. Nat. Rev. Neurol. 6 , 393-403. doi: 10.1038/nrneurol. 2010.74

Shulman, R. G., Rothman, D. L., Behar, K. L., and Hyder, F. (2004). Energetic basis of brain activity: implications for neuroimaging. Trends Neurosci. 27, 489-495. doi: 10.1016/j.tins.2004.06.005

Siakotos, A. N., Rouser, G., and Fleischer, S. (1969). Isolation of highly purified human and bovine brain endothelial cells and nuclei and their phospholipid composition. Lipids 4, 234-239. doi: 10.1007/BF02532638

Siddharthan, V., Kim, Y. V., Liu, S., and Kim, K. S. (2007). Human astrocytes/astrocyteconditioned medium and shear stress enhance the barrier properties of human brain microvascular endothelial cells. Brain Res. 1147, 39-50. doi: 10.1016/j.brainres. 2007.02.029 
Siflinger-Birnboim, A., Delvecchio, P. J., Cooper, J. A., Blumenstock, F. A., Shepard, J. M., and Malik, A. B. (1987). Molecular-sieving characteristics of the cultured endothelial monolayer. J. Cell. Physiol. 132, 111-117. doi: 10.1002/jcp.1041320115

Sims, D. E. (1986). The pericyte - a review. Tissue Cell 18, 153-174. doi: 10.1016/0040-8166(86)90026-1

Sloan, C. D. K., Nandi, P., Linz, T. H., Aldrich, J. V., Audus, K. L., and Lunte, S. M. (2012). "Analytical and biological methods for probing the blood-brain barrier," in Annual Review of Analytical Chemistry, Vol. 5, eds R. G. Cooks and E. S. Yeung (Palo Alto, CA: Annual Reviews), 505-531.

Smith, Q. R. (2003). "A review of blood-brain barrier transport techniques," in The Blood-Brain Barrier: Biology and Research Protocols, ed S. Nag (Totowa, NJ: Humana Press), 193-208.

Smith, Q. R., and Allen, D. D. (2003). "In situ brain perfusion technique," in The Blood-Brain Barrier: Biology and Research Protocols, ed. S. Nag (Totowa, NJ: Humana Press), 209-218.

Smith, Q. R., and Takasato, Y. (1986). Kinetics of amino acid transport at the blood-brain barrier studied using an in situ brain perfusion technique. Ann. N.Y. Acad. Sci. 481, 186-201. doi: 10.1111/j.17496632.1986.tb27150.x

Snodgrass, J. J., Leonard, W. R., and Robertson, M. L. (2009). The energetics of encephalization in early hominids. Evol. Hominin Diets 15-29. doi: 10.1007/978-1-40209699-0 2

Sohet, F., and Daneman, R. (2013). Genetic mouse models to study blood-brain barrier development and function. Fluids Barriers CNS 10, 3. doi: 10.1186/20458118-10-3

Stahel, P. F., Shohami, E., Younis, F. M., Kariya, K., Otto, V. I., Lenzlinger, P. M., et al. (2000). Experimental closed head injury: analysis of neurological outcome, blood-brain barrier dysfunction, intracranial neutrophil infiltration, and neuronal cell death in mice deficient in genes for proinflammatory cytokines. J. Cereb. Blood Flow Metab. 20, 369-380. doi: 10.1097/00004647-20000200000019

Stenberg, P., Norinder, U., Luthman, K., and Artursson, P. (2001). Experimental and computational screening models for the prediction of intestinal drug absorption.
J. Med. Chem. 44, 1927-1937. doi: 10.1021/jm001101a

Stins, M. F., Gilles, F., and Kim, K. S. (1997). Selective expression of adhesion molecules on human brain microvascular endothelial cells. J. Neuroimmunol. 76, 81-90. doi: 10.1016/S0165-5728 (97)00036-2

Summerfield, S. G., Read, K., Begley, D. J., Obradovic, T., Hidalgo, I. J., Coggon, S., et al. (2007). Central nervous system drug disposition: the relationship between in situ brain permeability and brain free fraction. J. Pharmacol. Exp. Ther. 322, 205-213. doi: 10.1124/jpet.107.121525

Summerfield, S. G., Stevens, A. J., Cutler, L., Del Carmen Osuna, M., Hammond, B., Tang, S.-P., et al. (2006). Improving the in vitro prediction of in vivo central nervous system penetration: integrating permeability, p-glycoprotein efflux, and free fractions in blood and brain. J. Pharmacol. Exp. Ther. 316, 1282-1290. doi: 10.1124/jpet.105.092916

Sykova, E., and Nicholson, C. (2008). Diffusion in brain extracellular space. Physiol. Rev. 88, 1277-1340. doi: 10.1152/physrev. 00027.2007

Taddei, A., Giampietro, C., Conti, A., Orsenigo, F., Breviario, F., Pirazzoli, V., et al. (2008). Endothelial adherens junctions control tight junctions by VEcadherin-mediated upregulation of claudin-5. Nat. Cell Biol. 10, 923-934. doi: 10.1038/ncb 1752

Takano, T., Tian, G. F., Peng, W., Lou, N., Libionka, W., Han, X., et al. (2006). Astrocyte-mediated control of cerebral blood flow. Nat. Neurosci. 9, 260-267. doi: 10.1038/nn1623

Takasato, Y., Rapoport, S. I., and Smith, Q. R. (1984). An in situ brain perfusion technique to study cerebrovascular transport in the rat. Am. J. Physiol. 247, H484-H493.

Tarbell, J. M. (2010). Shear stress and the endothelial transport barrier. Cardiovasc. Res. 87, 320-330. doi: $10.1093 / \mathrm{cvr} / \mathrm{cvq} 146$

Tewes, B. J., and Galla, H. J. (2001). Lipid polarity in brain capillary endothelial cells. Endothelium 8, 207-220. doi: $10.3109 / 10623320109051566$

Thanabalasundaram,

Schneidewind, J., Pieper, C., and Galla, H. J. (2011). The impact of pericytes on the blood-brain barrier integrity depends critically on the pericyte differentiation stage. Int. J. Biochem. Cell Biol. 43,
1284-1293. doi: 10.1016/j.biocel. 2011.05.002

Thorne, R. G., Hrabetova, S., and Nicholson, C. (2005). Diffusion measurements for drug design. Nat. Mater. 4, 713-713. doi $10.1038 /$ nmat 1489

Thorne, R. G., and Nicholson, C. (2006). In vivo diffusion analysis with quantum dots and dextrans predicts the width of brain extracellular space. Proc. Natl. Acad. Sci. U.S.A. 103, 5567-5572. doi: 10.1073/pnas.0509425103

Tilling, T., Engelbertz, C., Decker, S., Korte, D., Huwel, S., and Galla, H. J. (2002). Expression and adhesive properties of basement membrane proteins in cerebral capillary endothelial cell cultures. Cell Tissue Res. 310, 19-29. doi: 10.1007/s00441-002-0604-1

Tilling, T., Korte, D., Hoheisel, D., and Galla, H. J. (1998). Basement membrane proteins influence brain capillary endothelial barrier function in vitro. J. Neurochem. 71, 1151-1157. doi: 10.1046/j.14714159.1998.71031151.x

Toole, B. P. (2004). Hyaluronan: from extracellular glue to pericellular cue. Nat. Rev. Cancer 4, 528-539. doi: 10.1038/nrc1391

Tran, T. T., Mittal, A., Gales, T., Maleeff, B., Aldinger, T., Polli, J. W., et al. (2004). Exact kinetic analysis of passive transport across a polarized confluent MDCK cell monolayer modeled as a single barrier. J. Pharm. Sci. 93, 2108-2123. doi 10.1002/jps. 20105

Tsukita, S., and Furuse, M. (1999). Occludin and claudins in tightjunction strands: leading or supporting players? Trends Cell Biol. 9, 268-273. doi: 10.1016/S0962 8924(99)01578-0

Tsukita, S., Furuse, M., and Itoh, M. (2001). Multifunctional strands in tight junctions. Nat. Rev. Mol. Cell Biol. 2, 285-293. doi: $10.1038 / 35067088$

Tunblad, K., Jonsson, E. N., and Hammarlund-Udenaes, $\quad \mathrm{M}$ (2003). Morphine blood-brain barrier transport is influenced by probenecid co-administration. Pharm. Res. 20, 618-623. doi: 10.1023/A:1023250900462

Turitto, V. T. (1982). Blood viscosity, mass transport, and thrombogenesis. Prog. Hemost. Thromb. 6 , 139-177.

Turley, E. A., Noble, P. W., and Bourguignon, L. Y. (2002). Signaling properties of hyaluronan receptors. J. Biol. Chem. 277, 4589-4592. doi: 10.1074/jbc.R100038200
Uchiyama, S., Carlin, A. F., Khosravi, A., Weiman, S., Banerjee, A., Quach, D., et al. (2009). The surface-anchored NanA protein promotes pneumococcal brain endothelial cell invasion. J. Exp. Med. 206, 1845-1852. doi: 10.1084/jem.20090386

Ueno, M. (2009). Mechanisms of the penetration of blood-borne substances into the brain. Curr. Neuropharmacol. 7, 142-149. doi: 10.2174/157015909788848901

Ullian, E. M., Sapperstein, S. K., Christopherson, K. S., and Barres, B. A. (2001). Control of synapse number by glia. Science 291, 657-661. doi: 10.1126/science.291. 5504.657

Ungerstedt, U. (1991). Microdialysisprinciples and applications for studies in animals and man. J. Intern. Med. 230, 365-373. doi: 10.1111/j.13652796.1991.tb00459.x

Volterra, A., and Meldolesi, J. (2005). Astrocytes, from brain glue to communication elements: the revolution continues. Nat. Rev. Neurosci. 6 , 626-640. doi: 10.1038/nrn1722

Wang, X., Sykes, D. B., and Miller, D. S. (2010). Constitutive androstane receptor-mediated up-regulation of atp-driven xenobiotic efflux transporters at the blood-brain barrier. Mol. Pharmacol. 78, 376-383. doi: 10.1124/mol.110.063685

Waterhouse, R. N. (2003). Determination of lipophilicity and its use as a predictor of blood-brain barrier penetration of molecular imaging agents. Mol. Imaging Biol. 5, 376-389. doi: 10.1016/j.mibio.2003.09.014

Waubant, E. (2006). Biomarkers indicative of blood-brain barrier disruption in multiple sclerosis. Dis. Markers 22, 235-244. doi: 10.1155/2006/709869

Wen, H., Watry, D. D., Marcondes, M. C., and Fox, H. S. (2004). Selective decrease in paracellular conductance of tight junctions: role of the first extracellular domain of claudin-5. Mol. Cell. Biol. 24, 8408-8417. doi: $\quad$ 10.1128/MCB.24.19.8408 8417.2004

Wils, P., Warnery, A., Phungba, V., Legrain, S., and Scherman, D. (1994). High lipophilicity decreases drug transport across intestinal epithelial-cells. J. Pharmacol. Exp. Ther. 269, 654-658.

Winkler, E. A., Bell, R. D., and Zlokovic, B. V. (2011). Central nervous system pericytes in health and disease. Nat. Neurosci. 14, 1398-1405. doi: 10.1038/nn.2946 
Wolburg, H., and Lippoldt, A. (2002). Tight junctions of the blood-brain barrier: development, composition and regulation. Vascul. Pharmacol. 38, 323-337. doi: 10.1016/S15371891(02)00200-8

Yamaguchi, Y. (2000). Lecticans: organizers of the brain extracellular matrix. Cell. Mol. Life Sci. 57, 276-289. doi: 10.1007/PL00000690

Yee, S. Y. (1997). In vitro permeability across Caco2 cells (colonic) can predict in vivo (small intestinal) absorption in man - Fact or myth. Pharm. Res. 14, 763-766. doi: 10.1023/A:1012102522787

Youdim, K. A., Avdeef, A., and Abbott, N. J. (2003). In vitro trans-monolayer permeability calculations: often forgotten assumptions. Drug Discov. Today 8, 997-1003. doi: 10.1016/S1359-6446 (03)02873-3

Youdim, K. A., Qaiser, M. Z., Begley, D. J., Rice-Evans, C. A., and Abbott, N. J. (2004). Flavonoid permeability across an in situ model of the blood-brain barrier. Free Radic. Biol.
Med. 36, 592-604. doi: 10.1016/j. freeradbiomed.2003.11.023

Zhao, R., Kalvass, J. C., and Pollack, G. M. (2009). Assessment of bloodbrain barrier permeability using the in situ mouse brain perfusion technique. Pharm. Res. 26, 1657-1664. doi: 10.1007/s11095009-9876-4

Zhong, Z., Deane, R., Ali, Z., Parisi, M., Shapovalov, Y., O’banion, M. K., et al. (2008). ALS-causing SOD1 mutants generate vascular changes prior to motor neuron degeneration. Nat. Neurosci. 11, 420-422. doi: $10.1038 / \mathrm{nn} 2073$

Zhu, X. H., Qiao, H. Y., Du, F., Xiong, Q., Liu, X., Zhang, X. L., et al. (2012). Quantitative imaging of energy expenditure in human brain. Neuroimage 60, 2107-2117. doi: 10.1016/j.neuro image.2012.02.013

Zimmermann, D. R., and DoursZimmermann, M. T. (2008) Extracellular matrix of the central nervous system: from neglect to challenge. Histochem.
Cell Biol. 130, 635-653. doi: 10.1007/s00418-008-0485-9

Zipser, B. D., Johanson, C. E., Gonzalez, L., Berzin, T. M., Tavares, R. Hulette, C. M., et al. (2007). Microvascular injury and bloodbrain barrier leakage in Alzheimer's disease. Neurobiol. Aging 28, 977-986. doi: 10.1016/j.neuro biolaging.2006.05.016

Zlokovic, B. V. (2005). Neurovascular mechanisms of Alzheimer's neurodegeneration. Trends Neurosci. 28, 202-208. doi: 10.1016/j.tins.2005.02.001

Zonta, M., Angulo, M. C., Gobbo, S., Rosengarten, B., Hossmann, K. A. Pozzan, T., et al. (2003). Neuronto-astrocyte signaling is central to the dynamic control of brain microcirculation. Nat. Neurosci. 6 , 43-50. doi: 10.1038/nn980

Conflict of Interest Statement: The authors declare that the research was conducted in the absence of any commercial or financial relationships that could be construed as a potential conflict of interest.

Received: 02 April 2013; accepted: 07 August 2013; published online: 30 August 2013.

Citation: Wong $A D, Y e$, Levy $A F$, Rothstein JD, Bergles DE and Searson PC (2013) The blood-brain barrier: an engineering perspective. Front. Neuroeng. 6:7. doi: 10.3389/fneng.2013.00007

This article was submitted to the journal Frontiers in Neuroengineering.

Copyright (c) 2013 Wong, Ye, Levy, Rothstein, Bergles and Searson. This is an open-access article distributed under the terms of the Creative Commons Attribution License (CC BY). The use, distribution or reproduction in other forums is permitted, provided the original author(s) or licensor are credited and that the original publication in this journal is cited, in accordance with accepted academic practice. No use, distribution or reproduction is permitted which does not comply with these terms. 